TRANSACTIONS OF THE

AMERICAN MATHEMATICAL SOCIETY

Volume 352, Number 9 , Pages $4295-4323$

S 0002-9947(00)02493-4

Article electronically published on May 23, 2000

\title{
THE NOETHERIAN PROPERTY IN SOME QUADRATIC ALGEBRAS
}

\author{
XENIA H. KRAMER
}

\begin{abstract}
We introduce a new class of noncommutative rings called pseudopolynomial rings and give sufficient conditions for such a ring to be Noetherian. Pseudopolynomial rings are standard finitely presented algebras over a field with some additional restrictions on their defining relations-namely that the polynomials in a Gröbner basis for the ideal of relations must be homogeneous of degree 2-and on the Ufnarovskii graph $\Gamma(A)$. The class of pseudopolynomial rings properly includes the generalized skew polynomial rings introduced by $\mathrm{M}$. Artin and W. Schelter. We use the graph $\Gamma(A)$ to define a weaker notion of almost commutative, which we call almost commutative on cycles. We show as our main result that a pseudopolynomial ring which is almost commutative on cycles is Noetherian. A counterexample shows that a Noetherian pseudopolynomial ring need not be almost commutative on cycles.
\end{abstract}

\section{INTRODUCTION}

Determining whether or not certain classes of noncommutative rings are Noetherian has been the focus of much previous research, and no doubt this topic will continue to be of interest since it is one of the most basic questions one might ask about any new class of rings. In this paper, we will be concerned with quadratic algebras. Let $K$ be a field, $X$ a finite set, $K\langle X\rangle$ the free associative algebra generated by $X$ over $K$, and $I \subset K\langle X\rangle$ an ideal. We say that the algebra $A=K\langle X\rangle / I$ is quadratic if $I$ is generated by quadratic polynomials in $K\langle X\rangle$. The Noetherian property has been studied for several important classes of quadratic algebras, including skew polynomial rings, algebras of solvable type, and Sklyanin algebras. Algebras of solvable type are a broad class which includes commutative polynomial rings, iterated Ore extensions of $K$, and universal enveloping algebras of finite dimensional Lie algebras over $K$. We give a survey of these results in Section 5 . The object of study in this paper will be a new class of quadratic algebras called pseudopolynomial rings which will be formally defined in Section 6 (we give a sketch below). We will see that the class of pseudopolynomial rings properly includes the generalized skew polynomial rings introduced by M. Artin and W. Schelter in [AS]. Our major tools will be the theory of Gröbner bases in free associative algebras

Received by the editors October 8, 1997 .

2000 Mathematics Subject Classification. Primary 16P40; Secondary 16S15, 16S37.

Key words and phrases. Noncommutative Noetherian ring, standard finitely presented algebra, noncommutative Gröbner basis, quadratic algebra.

This paper was written as partial fulfillment of the requirements for the Ph.D. degree at New Mexico State University under the direction of R. Laubenbacher, who has the author's warmest gratitude. 
and a graph, introduced by V. Ufnarovskii [U1], which may be associated to a quotient of a free algebra (given a sufficiently nice presentation) and used to study the quotient algebra.

Gröbner bases were first introduced for ideals in commutative polynomial rings by $\mathrm{B}$. Buchberger $\mathrm{Bu}$, who also gave an algorithm for computing them. These bases have proved to be powerful computational tools in commutative algebra and algebraic geometry. That Buchberger's algorithm will indeed halt is guaranteed by the fact that commutative polynomial rings are Noetherian. One of the major difficulties in extending the theory of Gröbner bases and Buchberger's algorithm to noncommutative rings is that such rings are generally not Noetherian. Nonetheless, there is a theory of Gröbner bases for free associative algebras, due to G. Bergman B] and F. Mora [M1, [M4]. Theories of Gröbner bases for a number of other noncommutative settings are a subject of current research; in this context, being able to determine whether an algebra is Noetherian is helpful for developing a Gröbner basis theory for the algebra. The great power of a Gröbner basis $G$ of an ideal $I \subset K\langle X\rangle$ is that it gives us a means of determining a canonical representative in $K\langle X\rangle$ of an element in the quotient $K\langle X\rangle / I$ and of solving the ideal membership problem for $I$. That is, given an element $f \in K\langle X\rangle$, we can determine whether or not $f \in I$.

To give an idea of what a pseudopolynomial ring is, suppose that we have a finite set $G \subset K\langle X\rangle$, and we let $I$ be the ideal generated by $G$ in $K\langle X\rangle$. If $G$ is a Gröbner basis for $I$, then the quotient algebra $A=K\langle X\rangle / I$ is called standard finitely presented. To such an algebra $A$ we may associate a finite directed graph $\Gamma(A)$ which will reflect such properties of $A$ as the growth of $A$. Pseudopolynomial rings are standard finitely presented algebras with additional restrictions on relations in $I$ namely that the polynomials in $G$ must be homogeneous of degree 2 -and on the graph $\Gamma(A)$. So pseudopolynomial rings are examples of quadratic algebras. For a pseudopolynomial ring, the vertices of $\Gamma(A)$ are exactly the elements of $X$, and the collection of all paths in $\Gamma(A)$ (including the empty path) will give a vector space basis for $A$. We also require that there be no intersecting cycles in $\Gamma(A)$, and that the graph $\Gamma(A)$ looks like a string of $m$ cycles:

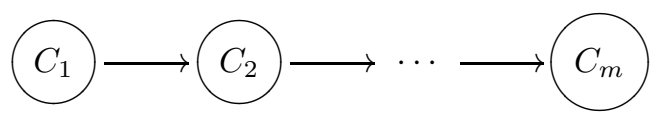

We allow multiple edges between cycles, and there may be edges from a cycle $C_{i}$ to a cycle $C_{j}$ as long as $i<j$; however, there may not be an edge from $C_{j}$ to $C_{i}$. The idea of pseudopolynomial rings is that we wish to view $A$ as being generated as an algebra by the cycles in $\Gamma(A)$, rather than the elements of $X$, as much as possible.

Recall that a finitely generated $K$-algebra $R$ is almost commutative if it has a filtration $\left\{R_{n}\right\}$ such that $R_{1}^{n}=R_{n}$ for each $n, R_{0}=K$, and $\operatorname{dim}_{K} R_{n}<\infty$, and the associated graded ring

$$
\operatorname{gr}(R)=\bigoplus_{n \geq 0} R_{n} / R_{n-1}
$$

is commutative. (Note that $R_{-1}=0$.) Almost commutative algebras are Noetherian, and are an interesting class in their own right, as an algebra is almost commutative if and only if it is a homomorphic image of the enveloping algebra of some finite dimensional Lie algebra over $K$ [MR, pp. 299-300]. If $\langle X\rangle$ is the free associative monoid generated by $X$, we may put a term ordering on $\langle X\rangle$. Using 
this term ordering, we may determine the set of leading monomials of $I$, denoted $\operatorname{LM}(I)$. Then the set $N=\langle X\rangle \backslash \operatorname{LM}(I)$ is a $K$-basis for $A$. For any $f \in K\langle X\rangle$, let $\operatorname{Nor}(f)$ be the image of $f$ in $A$. We may assume $\operatorname{Nor}(f) \in \operatorname{Span} N$, and denote the leading monomial of $\operatorname{Nor}(f)$ by $\bar{f}$. We say $A$ is almost commutative if for $u, v \in N$, we have $\overline{u v}=\overline{v u}$ GI1. If we consider the $N$-filtration $\left\{A_{u}: u \in N\right\}$ on $A$, where $A_{u}=\operatorname{Span}\{v \in N: v \leq u\}$, we may associate to $A$ an $N$-graded structure, which is commutative [GI1], [M3]. So this definition of almost commutative is a natural extension of the usual one. We will further extend this idea to a definition of almost commutative on cycles for pseudopolynomial rings $A$ : if $u, v \in N$ correspond to paths in different cycles in $\Gamma(A)$, then $\overline{u v}=\overline{v u}$. Our main result, Theorem 6.15, shows that if a pseudopolynomial ring is almost commutative on cycles, then it is Noetherian. The converse is false, however.

Finally, we note that given a standard finitely presented algebra $A=K\langle X\rangle / I$, one can effectively determine, by means of the graph $\Gamma(A)$, whether or not $A$ is indeed a pseudopolynomial ring. The Gröbner basis $G$ of $I$ allows us to draw the graph, and once we have $\Gamma(A)$, it is easy to determine the cycles of $\Gamma(A)$ and thus to check whether $A$ satisfies the conditions of a pseudopolynomial ring. From the graph, we can read off exactly what relations are needed for $A$ to be almost

commutative on cycles. We then see if these relations are in the ideal $I$. If so, we know $A$ is almost commutative on cycles, and hence, Noetherian.

\section{GRÖBNER BASES}

In this section, we establish notation and briefly review the theory of Gröbner bases in free associative algebras. A detailed treatment may be found in [M1] or M4.

Let $K$ be a field, $X$ a finite set, and $\langle X\rangle$ the free associative monoid generated by $X$. The elements of $X$ are called letters, and those of $\langle X\rangle$ are called words. The identity element 1 in $\langle X\rangle$ is the empty word (i.e., the word composed of no letters). For a subset $U \subset\langle X\rangle$, we write $\langle U\rangle$ for the monoid ideal generated by $U$ in $\langle X\rangle$. Let $K\langle X\rangle$ be the free associative algebra generated by $\langle X\rangle$ over $K$. For a subset $F \subset K\langle X\rangle$, the ideal generated by $F$ will be written $(F)$.

For any nonzero element $f \in K\langle X\rangle$, we define the leading monomial of $f$, denoted $\operatorname{LM}(f)$, to be the largest monomial (with respect to the ordering $<$ ) to appear in the representation of $f$ in the $K$-basis $\langle X\rangle$. The leading coefficient $\mathrm{LC}(f)$ of $f$ is the coefficient of $\operatorname{LM}(f)$, and the leading term $\operatorname{LT}(f)$ of $f$ is

$$
\mathrm{LT}(f)=\mathrm{LC}(f) \operatorname{LM}(f) .
$$

For $F \subseteq K\langle X\rangle$, we define

$$
\operatorname{LM}(F)=\{u \in\langle X\rangle: u=\operatorname{LM}(f) \text { for some } f \in F\} .
$$

For any (left, right, or two-sided) ideal $I \subset K\langle X\rangle$, we may determine the set

$$
\operatorname{LM}(I)=\{\operatorname{LM}(f) \in\langle X\rangle: f \in I\}
$$

which is a (left, right, two-sided) ideal of $\langle X\rangle$. We give the definition of a Gröbner basis for such an ideal $I$.

Definition 2.1. Let $I \subset K\langle X\rangle$ be an ideal. A set $F \subset I$ is a Gröbner basis for $I$ if the monoid ideal generated by $\operatorname{LM}(F)$ in $\langle X\rangle$ equals $\operatorname{LM}(I)$. That is,

$$
\langle\mathrm{LM}(F)\rangle=\mathrm{LM}(I) \text {; }
$$


or, equivalently,

$$
(\operatorname{LM}(F))=(\operatorname{LM}(I))
$$

as ideals in $K\langle X\rangle$.

A Gröbner basis $F$ is a generating set for the ideal $I$. Moreover, having this Gröbner basis allows us to find canonical representatives in $K\langle X\rangle$ of elements in the quotient $K\langle X\rangle / I$. Let $N(I)=\langle X\rangle \backslash \operatorname{LM}(I)$. (We will usually write $N(I)$ as just $N$.) Then

$$
K\langle X\rangle=I \oplus \operatorname{Span} N
$$

as vector spaces. So for all $f \in K\langle X\rangle$ [M1, [M4], there is a unique $g \in \operatorname{Span} N$ such that $f-g \in I$. We denote this $g$ by $\operatorname{Nor}(f)$ and call it the normal form of $f$. The element $\operatorname{Nor}(f) \in K\langle X\rangle$ is the canonical representation of the image of $f$ in the quotient $K\langle X\rangle / I$. We now note two simple, but important, facts:

(1) $f \in I$ if and only if $\operatorname{Nor}(f)=0$.

(2) For $u \in\langle X\rangle, \operatorname{Nor}(u)=u$ if and only if $u \in N$. Hence, the elements of $N$ are called the normal monomials of $K\langle X\rangle / I$.

Finally, we note that some Gröbner bases are better than others.

Definition 2.2. A Gröbner basis $G$ of an ideal $I \subset K\langle X\rangle$ is a reduced Gröbner basis if

1. $\{\operatorname{LM}(g): g \in G\}$ is an irredundant basis of $\operatorname{LM}(I)$,

2. $\mathrm{LC}(g)=1$ for each $g \in G$, and

3. $g=\operatorname{LM}(g)-\operatorname{Nor}(\operatorname{LM}(g))$ for each $g \in G$.

Any ideal $I \subset K\langle X\rangle$ has a unique reduced Gröbner basis [M4, Proposition 1.5]. If we have a finite Gröbner basis for $I$, it is then easy to find the reduced Gröbner basis for $I$.

\section{The UfNAROVSKII GRAPH}

Let $K$ be a field, $X$ a finite set, and let $\langle X\rangle$ be the free monoid generated by $X$. Let $K\langle X\rangle$ be the free associative algebra generated by $\langle X\rangle$ over $K$. For a finite set $G \subset K\langle X\rangle$, let $R=(G)$, the (two-sided) ideal generated by $G$ in $K\langle X\rangle$. Then the quotient, $A=K\langle X\rangle / R$, is a finitely presented algebra. If, in addition, the set $G$ forms a Gröbner basis for $R$, we say that $A$ is standard finitely presented (abbreviated s.f.p.). We will assume without loss of generality that $G$ is a reduced Gröbner basis.

As noted in Section 2, the set $N=\langle X\rangle \backslash \operatorname{LM}(R)$ forms a $K$-basis for $A$. Words in $N$ are called normal. Every subword of a normal word is normal. A word that is not in $N$ (so it is in $\operatorname{LM}(R)$ ), but whose proper subwords are normal, is called an obstruction. So the set of obstructions $F$ is just $\operatorname{LM}(G)$ (since $G$ is reduced). In [U1, Ufnarovskii shows how we may associate a finite directed graph $\Gamma(A)$ (which we will call the Ufnarovskii graph) to the algebra $A$ in such a way that the elements of $N$ are usefully reflected in the graph as paths.

A graph $\Gamma$ is a finite set of vertices $V$ joined by a finite set of edges $E$. We will assume that all graphs are directed. By a path of length $n$ in $\Gamma$, we mean an alternating sequence of vertices and edges $v_{0}, e_{1}, v_{1}, e_{2}, v_{2}, \ldots, v_{n-1}, e_{n}, v_{n}$ in which every edge $e_{i}$ begins at vertex $v_{i-1}$ and ends at vertex $v_{i}$. If $v_{0}=v_{n}$, the path is called cyclic; and the subgraph of $\Gamma$ consisting of the vertices $v_{i}, 0 \leq i<n$, and 
edges $e_{j}, 1 \leq j \leq n$, is called a cycle. If a vertex in $\Gamma$ is contained in some cycle, the vertex is cyclic; if the vertex is contained in no cycle, it is acyclic. A vertex which is contained in two distinct cycles is doubly cyclic.

In giving the construction of the graph $\Gamma(A)$, we will restrict our attention to the case in which $G$ consists only of homogeneous polynomials of degree 2 since this case includes all pseudopolynomial rings. (We will define pseudopolynomial rings in Section 6.) We construct the graph $\Gamma(A)$ in the following way: Let the set of vertices $V=X$. We draw an edge $e=x_{i} x_{j}$ from a vertex $x_{i}$ to a vertex $x_{j}$ if and only if $x_{i} x_{j} \notin(F)$. Thus there is a one-to-one correspondence between the edges in the graph $\Gamma(A)$ and the normal words of length 2. (Since $G$ is a Gröbner basis for $R$, we know that $(\operatorname{LM}(G))=(\operatorname{LM}(R))$ as ideals in $K\langle X\rangle$. So words not in $(F)=(\operatorname{LM}(G))$ are exactly the words in $N$.)

Example 3.1. Let $X=\{x, y, z, w\}$, and suppose

$$
F=\{x y, x w, y y, y z, y w, z x, z z, w x, w y, w z\} .
$$

So $\Gamma(A)$ is

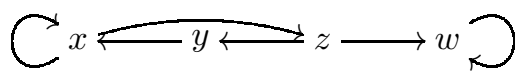

For instance, there is an edge from $x$ to $z$ since $x z \notin(F)$. There is no edge from $x$ to $y$ since $x y \in(F)$.

We may extend the one-to-one correspondence between edges and normal words of length 2 to a one-to-one correspondence between paths in the graph and normal words of length greater than or equal to 2 . Let $u \in N$ be such that the length of $u=m>1$. Write

$$
u=x_{1} x_{2} \cdots x_{m}
$$

where each $x_{i} \in X$. Then we may uniquely associate $u$ with the path of length $m-1$ in $\Gamma(A)$ through the successive edges

$$
x_{1} x_{2}, x_{2} x_{3}, \ldots, x_{m-1} x_{m} .
$$

Example 3.2. The word $y x x z w$ is normal in the algebra of Example 3.1. Since the length of $y x x z w$ is 5 , this word corresponds to a path of length 4 in $\Gamma$. This path passes through the edges $y x, x x, x z, z w$; that is, it is the path

$$
\begin{array}{r}
v_{0}=y, e_{1}=y x, v_{1}=x, e_{2}=x x, v_{2}=x, \\
e_{3}=x z, v_{3}=z, e_{4}=z w, v_{4}=w .
\end{array}
$$

\section{The growth of Algebras}

We first recall the notion of growth for monotone increasing functions. For monotone increasing functions $f, g: \mathbb{N} \rightarrow \mathbb{R}^{+}$, we say that $f \leq g$ if and only if there exist natural numbers $m, c>0$ such that $f(n) \leq c g(m n)$ for all $n \in \mathbb{N}$. Then we define an equivalence relation $\sim$ on such functions by

$$
f \sim g \text { if and only if } f \leq g \text { and } g \leq f .
$$

The equivalence class of a function $f$ is denoted $[f]$ and is called the growth of $f$. Growth $\left[2^{n}\right]$ is called exponential, and growth $\left[n^{d}\right]$ (where $d \in \mathbb{N}$ ) is called polynomial of degree $d$. 
To define the growth of an algebra, suppose $V$ is a finite dimensional vector space over $K$ which generates $A$ as an algebra. We define the function

$$
d_{V}(n)=\operatorname{dim}_{K}\left(K+V+V^{2}+\cdots+V^{n}\right)
$$

and take the growth of $A$ to be the equivalence class $\left[d_{V}(n)\right]$. The growth of $A$ is independent of the choice of $V$. For an extensive discussion of notion of growth in algebras, see $\mathrm{KL}$.

In [U1], Ufnarovskii shows that the growth of an s.f.p. algebra is either polynomial or exponential, and gives the following criterion in terms of the graph described in the previous section.

Theorem 4.1 ([U1]). Suppose $A$ is an s.f.p. algebra. Then the growth of $A$ is either polynomial or exponential. Furthermore:

(1) the growth of $A$ is exponential if and only if $\Gamma(A)$ has a doubly cyclic vertex (i.e., there are two intersecting cycles);

(2) the growth of $A$ is polynomial if and only if $\Gamma(A)$ is cyclically simple (i.e., there are no intersecting cycles). The polynomial degree $d$ is given by the maximal number of different cycles through which one path can pass.

Example 4.2. If $A=K\langle X\rangle /(F)$ where $X=\{x, y, z, w\}$, and

$$
F=\{x y, x w, y y, y z, y w, z x, z z, w x, w y, w z\}
$$

as in Example 3.1, then we know that $\Gamma(A)$ is

$$
C x \longleftarrow y \longleftarrow z \longrightarrow
$$

Since $x$ is a doubly cyclic vertex, the growth of $A$ is exponential. If we were to add the word $x z$ to $F$, then we would have

$$
C x \longleftarrow y \longleftarrow z \longrightarrow w \supset
$$

The growth would be polynomial of degree 1 . If we were to add $x x, x z$, and $w w$ to $F$, the graph would be

$$
x \longleftarrow y \longleftarrow z \longrightarrow w
$$

and the growth of $A$ would be polynomial of degree 0, i.e., constant. So $A$ would be finite dimensional.

\section{The Noetherian Property in QUAdratic algebras}

Let $A=K\langle X\rangle / I$ be a finitely presented algebra; if the defining relations in $I$ are all quadratic, we will call the algebra $A$ quadratic as well. In this section, we look at a variety of quadratic algebras and summarize results regarding the Noetherian property in these algebras. In the next section, we introduce a new class of quadratic algebras, which we call pseudopolynomial rings. Pseudopolynomial rings do not fit directly into any of the classes discussed below; however, we will see that the class of pseudopolynomial rings properly contains the generalized skew polynomial rings discussed by Artin and Schelter [AS].

An algebra $A=K\langle X\rangle / I$ for which $I$ is a monomial ideal (i.e., the defining relations in $I$ are all monomials) is called a monomial algebra. J. Okniński $[$ ] gives necessary and sufficient conditions for a monomial (not necessarily quadratic) algebra to be Noetherian. Let $M=\langle\operatorname{LM}(I)\rangle \subset\langle X\rangle$, and define the right multiplication graph of $\langle X\rangle / M$ to be the directed graph with the set of vertices $V=\langle X\rangle / M$ and 
an edge from a vertex $v$ to a vertex $w$ if and only if $v x=w$ for a letter $x \in X$. This graph is clearly a tree. Then we have the following result.

Proposition 5.1 $([\underline{\mathrm{O}})$. The algebra $K\langle X\rangle / I$ is right Noetherian if and only if the right multiplication graph of $\langle X\rangle / M$ has finitely many branchings.

Ufnarovskii shows in U2 that a monomial algebra is right Noetherian if and only if there are no edges coming out of any cycle of its graph $\Gamma(A)$. (Though the graphs used in U2 are somewhat different from those discussed in Sections 3 and 4 , the two types of graphs are equivalent with respect to this property.)

Regular algebras were introduced by Artin and Schelter in [AS]. Let $A=K \oplus$ $A_{1} \oplus A_{2} \oplus \cdots$ be a finitely presented graded algebra over a field $K$. The algebra $A$ is called regular if it has the following properties:

(1) $A$ has finite global dimension $d$ : every graded $A$-module has projective dimension $\leq d$.

(2) $A$ has polynomial growth.

(3) $A$ is Gorenstein; that is, $\operatorname{Ext}_{A}^{q}(k, A)=0$ for positive $q \neq d$, and $\operatorname{Ext}_{A}^{d}(k, A)$ $\cong k$.

Regular algebras of global dimension 3 are classified in [AS], where it is shown that if $A$ is regular of global dimension 3, then either $A$ is generated by two elements satisfying two cubic relations, or by three elements satisfying three quadratic relations. Artin, J. Tate and M. Van Den Bergh have shown that regular algebras of global dimension 3 are Noetherian [ATV]. A generalized notion of skew polynomial rings is also introduced in $\left[\mathrm{AS}\right.$. Let $X=\left\{x_{1}, x_{2}, \ldots, x_{n}\right\}$. Set a degreelexicographic (hereafter abbreviated deg-lex) term ordering with $\operatorname{deg} x_{i}=d_{i} \geq 1$ for $1 \leq i \leq n$, and $x_{1}<x_{2}<\cdots<x_{n}$. An ordered monomial is one of the form $x_{1}^{e_{1}} \cdots x_{n}^{e_{n}}$; let $T$ denote the set of all ordered monomials in $\langle X\rangle$.

Definition 5.2. An s.f.p. algebra $A=K\langle X\rangle / I$ is a skew polynomial ring if the reduced Gröbner basis $G$ of $I$ is of the form

$$
G=\left\{x_{j} x_{i}-\phi_{j i}: 1 \leq i<j \leq n\right\}
$$

where $\phi_{j i} \in \operatorname{Span} T$, the polynomial $\phi_{j i}$ is homogeneous of degree $d_{i}+d_{j}$, and $\operatorname{LM}\left(\phi_{j i}\right)<x_{j} x_{i}$.

Artin and Schelter note that skew polynomial rings may not be regular, and give a criterion for a regular algebra of global dimension 3 to be a skew polynomial ring AS.

A. Kandri-Rody and V. Weispfenning study algebras of solvable type and show that such algebras are Noetherian [KRW]. They define these algebras for a large class of term orderings on $\langle X\rangle$, but we will consider only the case where $<$ is a deg-lex ordering. Then we may make the following definition.

Definition 5.3. An s.f.p. algebra $A=K\langle X\rangle / I$ is an algebra of solvable type if the reduced Gröbner basis $G$ of $I$ is of the form

$$
G=\left\{x_{j} x_{i}-\alpha_{i j} x_{i} x_{j}-p_{i j}: 1 \leq i<j \leq n\right\}
$$

where $\alpha_{i j} \in K \backslash\{0\}, p_{i j} \in \operatorname{Span} T$, and $\operatorname{LM}\left(p_{i j}\right)<x_{i} x_{j}$.

If $p_{i j}$ is homogeneous of degree $d_{i}+d_{j}$, then clearly $A$ is also a skew polynomial ring. 
J. Apel [A] studies Gröbner bases in algebras $A$ to which graded rings $G$ with certain desirable properties may be associated. The algebras $A$ are termed $G$ algebras, and are defined as follows: let $A$ be a $K$-algebra and $(\Lambda,<)$ a well-ordered commutative Noetherian monoid. (We will indicate multiplication in $\Lambda$ by ०.) For technical reasons, we introduce an element $-\infty$ which satisfies $-\infty \circ \lambda=-\infty$ and $-\infty<\lambda$ for all $\lambda \in \Lambda$ and denote $\Lambda \cup\{-\infty\}$ by $\Lambda^{\infty}$. A function $\varphi: A \rightarrow \Lambda^{\infty}$ is a pseudovaluation of $A$ if it satisfies the following conditions:

(1) $(a+b)^{\varphi} \leq \max \left\{a^{\varphi}, b^{\varphi}\right\}$ for all $a, b \in A$;

(2) $(a b)^{\varphi} \leq a^{\varphi} \circ b^{\varphi}$ for all $a, b \in A$;

(3) $(\alpha a)^{\varphi}=a^{\varphi}$ for $\alpha \in K \backslash\{0\}$ and $a \in A$.

We can use such a pseudovaluation to define a filtration $F$ of $A$. For each $\lambda \in \Lambda^{\infty}$, let $F_{\lambda}=\left\{a \in A: a^{\varphi} \leq \lambda\right\}$ and $\hat{F}_{\lambda}=\bigcup_{\lambda^{\prime}<\lambda} F_{\lambda^{\prime}}$. Then let $G_{\lambda}=F_{\lambda} / \hat{F}_{\lambda}$, and define the algebra

$$
G=\bigoplus_{\lambda \in \Lambda} G_{\lambda}
$$

which is called the associated graded algebra of $A$. If an element $g \in G$ belongs to a single $G_{\lambda}$, then $g$ is called homogeneous of grade $\lambda$. For an arbitrary $g \in G$ such that $g \neq 0$, the grade of $g$ is the grade of its highest (with respect to $<$ ) homogeneous part. The grade of the zero element is $-\infty$. If $A$ satisfies the conditions:

(1) $\operatorname{dim}_{K} G_{\lambda} \leq 1$ for $\lambda \in \Lambda$ and

(2) $G_{\lambda} G_{\lambda^{\prime}}=G_{\lambda \circ \lambda^{\prime}}$ for $\lambda, \lambda^{\prime} \in \Lambda$,

then the quadruple $(A, \Lambda,<, \varphi)$ is called a $G$-algebra.

Apel shows that $G$-algebras are Noetherian. He notes that a commutative polynomial ring $K\left[x_{1}, \ldots, x_{n}\right]$ may be regarded as a $G$-algebra by setting $\Lambda=\mathbb{N}^{n}$, < any ordering on $\mathbb{N}^{n}$ which makes it a well-ordered monoid, and $\varphi$ the function which assigns to any nonzero polynomial the exponent vector of the leading monomial. Algebras of solvable type may be viewed as $G$-algebras in a similar way.

Mora mentions a result for another class of algebras in [M2]. These are finitely presented algebras $A=K\langle X\rangle / I$ in which the defining relations in $I$ are

$$
G=\left\{x_{j} x_{i}-\alpha_{i j} x_{i} x_{j}: 1 \leq i<j \leq n\right\}
$$

where $\alpha_{i j} \in K$. (We may have $\alpha_{i j}=0$.) Mora shows that the set $G$ is a Gröbner basis for I, and determines when $A$ will be Noetherian. Let

$$
V_{1}=\left\{x_{j}: \alpha_{i j} \neq 0 \text { for each } i<j\right\}
$$

and

$$
V_{2}=\left\{x_{j}: \alpha_{i j}=0 \text { for some } i<j\right\} .
$$

We may assume without loss of generality that $V_{1}=\left\{x_{1}, \ldots, x_{s}\right\}$ and $V_{2}=$ $\left\{x_{s+1}, \ldots, x_{n}\right\}$, by rearranging the variables if necessary.

Proposition 5.4 ([M2]). The algebra $K\langle X\rangle /(G)$ is Noetherian if and only if for each $x_{k} \in V_{2}$, for each $j>k$, we have $\alpha_{k j} \neq 0$.

Mora then uses this result to find finite Gröbner bases for certain ideals in the free algebra $K\langle X\rangle$. The ideals are those which contain, for each $i, j, i<j$, the relations

$$
x_{j} x_{i}-\alpha_{i j} x_{i} x_{j}-p_{i j}
$$


where $\alpha_{i j} \in K, p_{i j} \in \operatorname{Span} T$, and $\operatorname{LM}\left(p_{i j}\right)<x_{i} x_{j}$.

Strictly ordered algebras are studied by T. Gateva-Ivanova in GI1 and GI2. Before giving the definition, we establish some notation that will also be used in the next section. Let $X=\left\{x_{1}, x_{2}, \ldots, x_{n}\right\}$. First, we fix the notation $<_{0}$ to be the deg-lex term ordering on $X$, where $\operatorname{deg} x_{i}=1$ for $1 \leq i \leq n$, and

$$
x_{1}<_{0} x_{2}<_{0} \cdots<_{0} x_{n} .
$$

Let $I \subset K\langle X\rangle$ be an ideal. Then for $f \in K\langle X\rangle$, we will denote the largest monomial in the normal form of $f$ by $\bar{f}$; i.e.,

$$
\bar{f}=\operatorname{LM}(\operatorname{Nor}(f)) .
$$

Now for $f, g \in K\langle X\rangle$, we define

$$
f \sim g \text { if and only if } \bar{f}=\bar{g} .
$$

(If $f \in I$, then we write $\bar{f}=0$ and $f \sim 0$.) Clearly $\sim$ gives an equivalence relation on $K\langle X\rangle$. We also define a partial order on $K\langle X\rangle$ as follows:

$$
f<g \text { if and only if } \bar{f}<_{0} \bar{g}
$$

for $\bar{f}, \bar{g} \in K\langle X\rangle \backslash\{0\}$, and $f>0$ for $\bar{f} \in K\langle X\rangle \backslash\{0\}$.

Definition 5.5 (GI1]). An algebra $A=K\langle X\rangle / I$ is strictly ordered if

$$
\operatorname{deg}\left(\overline{x_{i} x_{j}}\right)=2 \text { for } 1 \leq i, j \leq n,
$$

and if for $u, v, w \in N(I)$ such that $v<w$, we have $u v<u w$ and $v u<w u$.

Note that the second condition implies that strictly ordered algebras have no zero divisors.

Definition 5.6. An algebra $A=K\langle X\rangle / I$ is almost commutative if for any $u, v \in$ $N(I)$, we have $u v \sim v u$.

Gateva-Ivanova proves the following theorem in GI1, GI2.

Theorem 5.7. Let $I \subset K\langle X\rangle$ be an ideal generated by homogeneous elements. If $A=K\langle X\rangle / I$ is a strictly ordered s.f.p. algebra, then the following conditions are equivalent:

(1) A is almost commutative;

(2) A has polynomial growth;

(3) $A$ is right Noetherian;

(4) $A$ is left Noetherian;

(5) The reduced Gröbner basis $G$ of I has the following property: for any pair of integers $i, j, 1 \leq i \leq j \leq n$, either

(a) there is a polynomial $f_{j i} \in G$ such that

$$
f_{j i}=x_{j} x_{i}-\alpha_{j i} x_{i} x_{j}-p_{j i}
$$

where $\alpha_{j i} \in K \backslash\{0\}, p_{j i} \in$ Span $N$, and $\left(\right.$ if $\left.p_{j i} \neq 0\right) L M\left(p_{j i}\right)<_{0} x_{i} x_{j}$; or

(b) there are two polynomials $f_{j i}, f_{i j} \in F$ such that

$$
\begin{aligned}
& f_{j i}=x_{j} x_{i}-\alpha_{j i} x_{s} x_{t}-p_{j i}, \\
& f_{i j}=x_{i} x_{j}-\alpha_{i j} x_{s} x_{t}-p_{i j}
\end{aligned}
$$

where $s<i, t>j, \alpha_{j i}, \alpha_{i j} \in K \backslash\{0\}, p_{j i}, p_{i j} \in \operatorname{Span} N$, and $L M\left(p_{j i}\right)<_{0}$ $x_{s} x_{t}, L M\left(p_{i j}\right)<_{0} x_{s} x_{t}$. 
In [GI3], Gateva-Ivanova considers binomial skew polynomial rings, which are skew polynomial rings (as defined above) in which the reduced Gröbner basis $G$ is of the form

$$
G=\left\{x_{j} x_{i}-\alpha_{i j} x_{i^{\prime}} x_{j^{\prime}}: 1 \leq i<j \leq n\right\}
$$

where $\alpha_{i j} \in K \backslash\{0\}, 1 \leq i^{\prime} \leq j^{\prime} \leq n, x_{i^{\prime}} x_{j^{\prime}}<_{0} x_{j} x_{i}$.

Under the further assumption that $i^{\prime}<j^{\prime}$, thus excluding the case $i^{\prime}=j^{\prime}$, the following theorem is obtained.

Theorem 5.8. Let $A=K\left\langle x_{1}, \ldots, x_{n}\right\rangle /(G)$ be a binomial skew polynomial ring without zero divisors, with reduced Gröbner basis

$$
G=\left\{x_{j} x_{i}-\alpha_{i j} x_{i^{\prime}} x_{j^{\prime}}: 1 \leq i<j \leq n\right\},
$$

where for $1 \leq i<j \leq n$ one has $1 \leq i^{\prime}<j^{\prime} \leq n$, and $x_{i^{\prime}} x_{j^{\prime}}<_{0} x_{j} x_{i}$. Then the following conditions are equivalent:

(1) The set $\left\{x_{i^{\prime}} x_{j^{\prime}}: 1 \leq i<j \leq n\right\}$ contains precisely $\left(\begin{array}{c}n \\ 2\end{array}\right)$ elements;

(2) A is left Noetherian;

(3) A is right Noetherian.

In GI4, the restriction that $A$ have no zero divisors is removed, and finite Gröbner bases are found for any left or right ideal of $A$.

The Sklyanin algebra $S$ studied by S. Smith and J. Stafford SmSt] is another example of a quadratic algebra, and may be defined as follows: Let $\alpha, \beta, \gamma \in K$ satisfy

$$
\alpha+\beta+\gamma+\alpha \beta \gamma=0
$$

Then the algebra $S$ is given by $K\left\langle x_{1}, x_{2}, x_{3}, x_{4}\right\rangle /(F)$, where

$$
F=\left\{f_{1}, f_{2}, f_{3}, f_{4}, f_{5}, f_{6}\right\},
$$

and

$$
\begin{aligned}
& f_{1}=x_{1} x_{2}-x_{2} x_{1}-\alpha\left(x_{3} x_{4}+x_{4} x_{3}\right), \\
& f_{2}=x_{1} x_{2}+x_{2} x_{1}-\left(x_{3} x_{4}-x_{4} x_{3}\right), \\
& f_{3}=x_{1} x_{3}-x_{3} x_{1}-\beta\left(x_{4} x_{2}+x_{2} x_{4}\right), \\
& f_{4}=x_{1} x_{3}+x_{3} x_{1}-\left(x_{4} x_{2}-x_{2} x_{4}\right), \\
& f_{5}=x_{1} x_{4}-x_{4} x_{1}-\gamma\left(x_{2} x_{3}+x_{3} x_{2}\right), \\
& f_{6}=x_{1} x_{4}+x_{4} x_{1}-\left(x_{2} x_{3}-x_{3} x_{2}\right) .
\end{aligned}
$$

It is shown in [SmSt] that with some restrictions on $\alpha, \beta, \gamma$, these algebras are regular (in the sense of [AS]) and are Noetherian domains.

\section{Pseudopolynomial Rings}

Let $X=\left\{x_{1}, x_{2}, \ldots, x_{n}\right\}$. As in the previous section, we will use the notation $<_{0}$ for the deg-lex term ordering such that $\operatorname{deg} x_{i}=1$ for $1 \leq i \leq n$, and

$$
x_{1}<_{0} x_{2}<_{0} \cdots<_{0} x_{n} .
$$

This term ordering will be the only one used for the rest of the paper. Recall that given an ideal $I$ of $K\langle X\rangle$, we define

$$
\bar{f}=\operatorname{LM}(\operatorname{Nor}(f)),
$$


for $f \in K\langle X\rangle$, and the relations

$$
\begin{aligned}
& f \sim g \text { if and only if } \bar{f}=\bar{g}, \\
& f<g \text { if and only if } \bar{f}<_{0} \bar{g}
\end{aligned}
$$

for $f, g \in K\langle X\rangle$.

In the discussion that follows, we consider s.f.p. algebras $A=K\langle X\rangle / R$ with polynomial growth. For such an algebra $A$, the Ufnarovskii graph $\Gamma(A)$ must be cyclically simple; so we define a partial order on cycles in $\Gamma$ as follows: $C_{1} \leq C_{2}$ if there is a path in $\Gamma$ which originates at a vertex in $C_{1}$ and terminates at a vertex in $C_{2}$. If there are any acyclic vertices in $\Gamma$, we regard them as cycles of length 0 . We saw in Section 3 that for any $u \in N$ (where the length of $u$ is greater than or equal to 2 ), we may associate to $u$ a unique path in $\Gamma(A)$. We say that the monomial $u$ corresponds to a path in the cycle $C$ if all vertices and edges of the path we associate to $u$ lie in a single cycle $C$ in $\Gamma(A)$.

We further restrict the algebras we consider to those that satisfy the following definition.

Definition 6.1. An s.f.p. algebra $A=K\langle X\rangle / R$ with polynomial growth is called a pseudopolynomial ring if it satisfies the following conditions:

(1) The defining relations in $R$ are homogeneous of degree 2 .

(2) The partial order on cycles in $\Gamma$ is a total order which is compatible with the term ordering $<_{0}$; i.e., for two distinct cycles $C_{i}, C_{j}$, if $C_{i} \leq C_{j}$, then for any monomial $u$ corresponding to a path in $C_{i}$ and monomial $v$ corresponding to a path in $C_{j}$ such that $\operatorname{deg} u=\operatorname{deg} v$, we have $u<_{0} v$.

(3) If $x_{i}, x_{j}$ are vertices in distinct cycles such that $i<j$, then $x_{i} x_{j} \in N$.

(4) Suppose $v, w \in N$ correspond to paths in the same cycle $C$ in $\Gamma$ and $v w \notin N$. Then $\overline{v w}=0$.

Suppose $A=K\langle X\rangle / R$ is a pseudopolynomial ring. Since the defining relations in $R$ are quadratic (by condition (1)), the vertices of the Ufnarovskii graph of $A$ are exactly the elements of $X$; i.e., $V=X$. So for any path $v_{0}, e_{1}, v_{1}, \ldots, v_{t-1}, e_{t}, v_{t}$ in $\Gamma(A)$, the vertices $v_{i}$ are just letters in $X$, and the path corresponds to the monomial $v_{0} v_{1} \cdots v_{t}$ in $N$. From now on, we will use the notation

$$
v_{0} \rightarrow v_{1} \rightarrow \cdots \rightarrow v_{t}
$$

to indicate this path. Similarly, if we have a cycle in $\Gamma(A)$ consisting of the vertices $v_{0}, v_{1}, \ldots, v_{t}$ and the edges $e_{1}, e_{2}, \ldots, e_{t}$, then we will use the notation

$$
\left(v_{0} \rightarrow v_{1} \rightarrow \cdots \rightarrow v_{t} \rightarrow v_{0}\right)
$$

to indicate this cycle (which is a subgraph of $\Gamma$ ).

Since the defining relations in $R$ are also homogeneous, if we have $u, v \in N$ such that $\overline{u v} \neq 0$, then $\operatorname{Nor}(u v)$ is homogeneous of degree $(\operatorname{deg} u+\operatorname{deg} v)$. So the algebra $A$ is graded. Let $U_{i}=\operatorname{Span}\{u \in N: \operatorname{deg} u \leq i\}$; then

$$
U_{0} \subset U_{1} \subset \cdots \subset U_{i} \subset \cdots
$$

gives a filtration. So

$$
A=\bigoplus_{i \geq 0} A_{i}
$$


where $A_{0}=K$ and

$$
A_{i}=U_{i} / U_{i-1} \text { for } i>0 .
$$

Since by condition (2), we have a total order on cycles, let us assume that the cycles are numbered in such a way that if $i<j$, we have $C_{i}<C_{j}$. Any letter $x_{i} \in X$ may be viewed as a monomial corresponding to the path consisting of the single vertex $x_{i}$ in a cycle $C_{i}$ of $\Gamma$. Thus, if $C_{i}, C_{j}$ are cycles in $\Gamma$ such that $i<j$, then for any vertex $y$ of $C_{i}$ and $z$ of $C_{j}$, we must have $y<_{0} z$. Similarly, if $y, z$ are vertices in the same cycle, condition (4) says that either $y z \in N$ or $\overline{y z}=0$.

Example 6.2. Let $X=\{x, y, z\}$, and let $G=\left\{z^{2}, y^{2}, z x-x y, y x-x z\right\}$. Then the algebra $A=K\langle X\rangle /(G)$ is a pseudopolynomial ring. It is easily checked that $G$ is a Gröbner basis for $(G)$. (We assume $x<_{0} y<_{0} z$.) So the set of obstructions is $\operatorname{LM}(G)=\left\{z^{2}, y^{2}, z x, y x\right\}$, and $\Gamma(A)$ is

$$
\mathrm{C} \mathrm{\longrightarrow} \rightleftharpoons z
$$

Here the cycles are $C_{1}=(x \rightarrow x)$ and $C_{2}=(y \rightarrow z \rightarrow y)$.

If we let $F=\{z x-x y, y x-x z\}$, then $F$ is a Gröbner basis for $(F)$, but the algebra $B=K\langle X\rangle /(F)$ is not a pseudopolynomial ring. Its graph $\Gamma(B)$ is

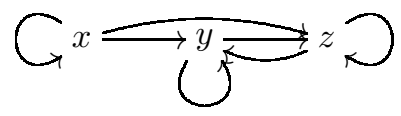

We see that $\Gamma(B)$ has two doubly cyclic vertices: $y$ and $z$. Hence, $B$ has exponential growth and cannot be a pseudopolynomial ring.

Example 6.3. Let $X=\left\{x_{1}, x_{2}, x_{3}, x_{4}\right\}$, and let

$$
G=\left\{x_{1}^{2}, x_{2}^{2}, x_{3}^{2}, x_{3} x_{1}-x_{2} x_{3}, x_{3} x_{2}-x_{1} x_{3}, x_{4} x_{1}-x_{2} x_{4}, x_{4} x_{2}-x_{1} x_{4}, x_{4} x_{3}-x_{3} x_{4}\right\} .
$$

Then the algebra $A=K\langle X\rangle /(G)$ is a pseudopolynomial ring, and $G$ is a Gröbner basis for $R$. The set of obstructions is

$$
\operatorname{LM}(G)=\left\{x_{1}^{2}, x_{2}^{2}, x_{3}^{2}, x_{3} x_{1}, x_{3} x_{2}, x_{4} x_{1}, x_{4} x_{2}, x_{4} x_{3}\right\} .
$$

So $\Gamma(A)$ is

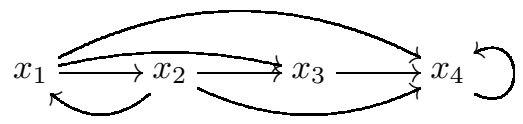

The cycles are $C_{1}=\left(x_{1} \rightarrow x_{2} \rightarrow x_{1}\right), C_{2}=\left(x_{3}\right)$ (a cycle of length 0 ), and $C_{3}=\left(x_{4} \rightarrow x_{4}\right)$.

It is fairly easy to create examples of pseudopolynomial rings. Start with a graph $\Gamma(A)$ that satisfies $(2)$ and (3): say let $\Gamma(A)$ be

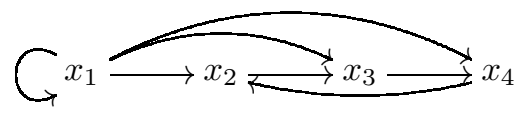

So the cycles are $C_{1}=\left(x_{1} \rightarrow x_{1}\right)$ and $C_{2}=\left(x_{2} \rightarrow x_{3} \rightarrow x_{4} \rightarrow x_{2}\right)$. Since there are four vertices in the graph, we put $X=\left\{x_{1}, x_{2}, x_{3}, x_{4}\right\}$. We now choose the set $G$ of generating relations for $R$ in such a way that $G$ forms a Gröbner basis for $R$ and conditions (1) and (4) are satisfied. Condition (4) requires that we put 
the monomials $x^{2}, x^{3}, x^{4}, x_{3} x_{2}, x_{4} x_{3}$, and $x_{4} x_{2}$ into $G$. Now we must choose homogeneous polynomials with leading terms $x_{2} x_{1}, x_{3} x_{1}$, and $x_{4} x_{1}$. An easy way is just to add these monomials to $G$. Otherwise, we must be careful that our choice results in $G$ being a Gröbner basis for $R$. The polynomials $x_{2} x_{1}-x_{1} x_{3}, x_{3} x_{1}-x_{1} x_{4}$, and $x_{4} x_{1}-x_{1} x_{2}$ will work.

Skew polynomial rings are examples of pseudopolynomial rings. For a skew polynomial ring $A=K\langle X\rangle / R$, we know that the reduced Gröbner basis $G$ of $R$ is of the form

$$
G=\left\{x_{j} x_{i}-\phi_{j i}: 1 \leq i<j \leq n\right\}
$$

where $\phi_{j i} \in \operatorname{Span} T$, the polynomial $\phi_{j i}$ is homogeneous of degree 2 (since we are assuming the term ordering $\left.<_{0}\right)$, and $\operatorname{LM}\left(\phi_{j i}\right)<x_{j} x_{i}$. The Ufnarovskii graph $\Gamma(A)$ is most easily expressed in terms of an adjacency matrix $M(\Gamma)=\left(a_{i j}\right)$, where $a_{i j}=1$ if and only if there is an edge from the vertex $x_{i}$ to the vertex $x_{j}$. Otherwise, $a_{j i}=0$. Since $\operatorname{LM}(G)=\left\{x_{j} x_{i}: 1 \leq i<j \leq n\right\}$, we have

$$
M(\Gamma)=\left(\begin{array}{ccccc}
1 & 1 & & \ldots & 1 \\
0 & 1 & & \ldots & 1 \\
\vdots & 0 & 1 & \ldots & \vdots \\
& \vdots & & \ddots & \\
0 & 0 & & \ldots & 1
\end{array}\right) .
$$

That is, $M(\Gamma)$ has 1's on and above the main diagonal and 0's below the main diagonal. The 1's on the diagonal mean that we have $n$ cycles of length 1: $C_{i}=$ $\left(x_{i} \rightarrow x_{i}\right)$ for each $i, 1 \leq i \leq n$. The graph $\Gamma$ is cyclically simple since the matrix entry $a_{i j}=0$ for $i>j$. If $u, v$ correspond to paths in cycles $C_{i}, C_{j}$, respectively, with $i<j$ and $\operatorname{deg} u=\operatorname{deg} v$, then $u=x_{i}^{k}$ and $v=x_{j}^{k}$ for some $k>0$. Clearly $u<_{0} v$; so condition (2) is satisfied. Since $a_{i j}=1$ for $i<j$, condition (3) is satisfied. If $u, v \in N$ correspond to paths in the same cycle $C_{i}$, the $u=x_{i}^{k}$ and $v=x_{i}^{l}$ for some $k, l>0$. So $u v=x_{i}^{k+l} \in N$, and we see that (4) is satisfied. Thus, skew polynomial rings are examples of pseudopolynomial rings. Pseudopolynomial rings which are not skew polynomial rings are shown in Examples 6.2 and 6.3.

Remark 6.4. If $A$ is a pseudopolynomial ring with monomials $u, v, w \neq 1$ such that $u v, v w \in N$, then $u v w \in N$. Since none of $u, v, w$ is the empty word, we have $\operatorname{deg} u, \operatorname{deg} v, \operatorname{deg} w \geq 1$, which implies that $\operatorname{deg} u v, \operatorname{deg} v w \geq 2$. Now suppose $u v w \notin N$, that is, uvw contains some obstruction $f$. By 1 , we know that $\operatorname{deg} f=2$; so either $f$ is a subword of $u v$ or $f$ is a subword of $v w$. But both $u v, v w \in N$, are contradictions. So we must have $u v w \in N$.

Definition 6.5. A pseudopolynomial ring $A=K\left\langle x_{1}, \ldots, x_{n}\right\rangle / R$ is almost commutative on cycles if for vertices $x_{i}, x_{j}$ from distinct cycles in $\Gamma(A)$, we have

$$
x_{j} x_{i} \sim x_{i} x_{j} .
$$

Remark 6.6. If $x_{i}, x_{j}$ are vertices in distinct cycles $C_{i}, C_{j}$, respectively, of $\Gamma(A)$ for some pseudopolynomial ring $A$ with $i<j$, then condition (2) implies that $x_{j} x_{i} \notin N$. Since $i<j$ implies that $C_{i}<C_{j}$ (since we have a total ordering on cycles) and $\Gamma$ is cyclically simple, there cannot be a path from $C_{j}$ to $C_{i}$. If $x_{j} x_{i}$ were normal, the edge from $x_{j}$ to $x_{i}$ would be such a path. Hence, $x_{j} x_{i} \notin N$. But $x_{i} x_{j} \in N$ by 
condition (3); so if $A$ is almost commutative on cycles, we have

$$
\overline{x_{j} x_{i}}=x_{i} x_{j} .
$$

Example 6.7. Let $X=\{x, y, z\}$ (assume $x<_{0} y<_{0} z$ ), and let

$$
G=\left\{z^{2}, y^{2}, y x-x y, z x-x z\right\} .
$$

Then if $A=K\langle x, y, z\rangle / R$ where $R=(G)$, it is easily checked that $G$ is a Gröbner basis for $R$. The graph $\Gamma(A)$ is

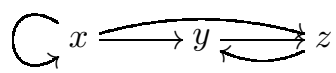

So, as in Example 6.2 , the cycles are $C_{1}=(x \rightarrow x)$ and $C_{2}=(y \rightarrow z \rightarrow y)$, and $A$ is clearly a pseudopolynomial ring but not a skew polynomial ring. For $A$ to be almost commutative on cycles, we need the vertex $x$ of $C_{1}$ to almost commute with both vertices, $y, z$, of $C_{2}$; i.e., we need

$$
\begin{aligned}
& \overline{y x}=x y, \\
& \overline{z x}=x z .
\end{aligned}
$$

These conditions are clearly satisfied since the relations $y x-x y$ and $z x-x z$ are in $R$. Notice that $A$ is not almost commutative since $y z \not z y$. Also, it is easy to see that neither the algebra of Example 6.2 nor that of Example 6.3 are almost commutative on cycles.

If an algebra $A=K\left\langle x_{1}, \ldots, x_{n}\right\rangle / R$ is a skew polynomial ring, the cycles in $\Gamma(A)$ are exactly $C_{i}=\left(x_{i} \rightarrow x_{i}\right)$ for each $i, 1 \leq i \leq n$. So a skew polynomial ring is almost commutative on cycles if and only if it is almost commutative.

Lemma 6.8 is a technical lemma needed to prove Lemma 6.9, which tells us the sense in which we may think of cycles in the Ufnarovskii graph as almost commuting.

Lemma 6.8. Let $A=K\left\langle x_{1}, x_{2}, \ldots, x_{n}\right\rangle / R$ be a pseudopolynomial ring which is almost commutative on cycles. Suppose $u \in N$ corresponds to a path in a cycle $C_{i}$ in $\Gamma$ and $x_{l} \in\left\{x_{1}, x_{2}, \ldots, x_{n}\right\}$ is a vertex in a cycle $C_{j}$ where $i<j$. Then $\overline{x_{l} u}=u x_{l} \in N$.

Proof. We proceed by induction on $\operatorname{deg} u$.

If $\operatorname{deg} u=1$, then $u=x_{k}$, where $x_{k} \in\left\{x_{1}, \ldots, x_{n}\right\}$. Since $i<j$, we have $x_{k}<x_{l}$; so $\overline{x_{l} u}=\overline{x_{l} x_{k}}=x_{k} x_{l} \in N$ by Remark 6.6.

If $\operatorname{deg} u=m>1$, then write $u=x_{k_{1}} \cdots x_{k_{m}}$, where each $x_{k_{p}} \in\left\{x_{1}, \ldots, x_{n}\right\}$. Since $i<j$, we have $x_{k_{p}}<_{0} x_{l}$ for $1 \leq p \leq m$. Then

$$
\begin{aligned}
\overline{x_{l} u} & =\overline{x_{l}\left(x_{k_{1}} \cdots x_{k_{m}}\right)} \\
& =\overline{\overline{\left(x_{l} x_{k_{1}}\right)\left(x_{k_{2}} \cdots x_{k_{m}}\right)}} \\
& =\overline{\left(x_{k_{1}} x_{l}\right)\left(x_{k_{2}} \cdots x_{k_{m}}\right)} \\
& =\overline{x_{k_{1}} \overline{\left(x_{l}\left(x_{k_{2}} \cdots x_{k_{m}}\right)\right)}} \\
& =\overline{x_{k_{1}}\left(\left(x_{k_{2}} \cdots x_{k_{m}}\right) x_{l}\right)} \text { by Remark } 6.6 \text { the induction hypothesis. }
\end{aligned}
$$

We also have that $x_{k_{2}} \cdots x_{k_{m}} x_{l} \in N$ by the induction hypothesis, and we know that $x_{k_{1}} \cdots x_{k_{m}}=u \in N$. So $x_{k_{1}} \cdots x_{k_{m}} x_{l} \in N$ by Remark 6.4 ; thus, $\overline{x_{l} u}=$ $x_{k_{1}} \cdots x_{k_{m}} x_{l}=u x_{l} \in N$. 
Lemma 6.9. Let $A=K\left\langle x_{1}, \ldots, x_{n}\right\rangle / R$ be a pseudopolynomial ring which is almost commutative on cycles. Suppose $u \in N$ corresponds to a path in a cycle $C_{i}$ in $\Gamma(A)$ and $v \in N$ corresponds to a path in a cycle $C_{j}$ where $i<j$. Then $\overline{v u}=u v \in N$.

Proof. We proceed by induction on $\operatorname{deg} v$.

If $\operatorname{deg} v=1$, then $v=x_{l}$ where $x_{l} \in\left\{x_{1}, \ldots, x_{n}\right\}$. We have shown that $\overline{v u}=$ $\overline{x_{l} u}=u x_{l} \in N$ in Lemma 6.8.

If $\operatorname{deg} v=m>1$, then write $v=x_{l_{1}} \cdots x_{l_{m}}$, where each $x_{l_{p}} \in\left\{x_{1}, \ldots x_{n}\right\}$. Note that each $x_{l_{p}}$ is a vertex in $C_{j}$ by assumption. Then

$$
\begin{aligned}
\overline{v u} & =\overline{\left(x_{l_{1}} \cdots x_{l_{m}}\right) u} \\
& =\overline{\left(x_{l_{1}} \cdots x_{l_{(m-1)}}\right) \overline{x_{l_{m}} u}} \\
& =\overline{\left(x_{l_{1}} \cdots x_{l_{(m-1)}}\right) u x_{l_{m}}} \\
& =\overline{\overline{\left.\left(x_{l_{1}} \cdots x_{l_{(m-1)}}\right) u\right)} x_{l_{m}}} \\
& =\overline{u\left(x_{l_{1}} \cdots x_{l_{(m-1)}}\right) x_{l_{m}}} \text { by the induction hypothesis. } 6.8
\end{aligned}
$$

Note that the induction hypothesis also tells us that $u x_{l_{1}} \cdots x_{l_{(m-1)}} \in N$, and we already know that $x_{l_{1}} \cdots x_{l_{m}}=v \in N$. So $u x_{l_{1}} \cdots x_{l_{m}} \in N$, and we have $\overline{v u}=u x_{l_{1}} \cdots x_{l_{m}}=u v \in N$.

Lemma 6.10 tells us to what extent the relations in a pseudopolynomial ring $K\langle X\rangle / R$ which is almost commutative on cycles are compatible with the term ordering on $\langle X\rangle$.

Lemma 6.10. Let $A=K\left\langle x_{1}, \ldots, x_{n}\right\rangle / R$ be a pseudopolynomial ring. Then if $A$ is almost commutative on cycles, A satisfies the following condition: If $u, v, w \in N$ such that $v<w$, then

(1) $\overline{u w} \neq 0$ implies $u v<u w$, and

(2) $\overline{w u} \neq 0$ implies $v u<w u$.

Proof. (1) Suppose $\overline{u w} \neq 0$. If $\overline{u v}=0$, then clearly $u v<u w$; so we may assume that $\overline{u v} \neq 0$. If $\operatorname{deg} v<\operatorname{deg} w$, then since $A$ is graded, we have

$$
\operatorname{deg}(\overline{u v})=\operatorname{deg} u+\operatorname{deg} v<\operatorname{deg} u+\operatorname{deg} w=\operatorname{deg}(\overline{u w}) .
$$

So $u v<u w$.

Suppose $\operatorname{deg} v=\operatorname{deg} w$. We know that $v<_{0} w$; the idea here is to look at $v$ and $w$ as products of cycles in the Ufnarovskii graph $\Gamma(A)$ and compare them cycle by cycle. Say $\Gamma(A)$ has $m$ distinct cycles and the path corresponding to $w$ originates in $C_{j}$. Then we may write

$$
w=w_{j} w_{j+1} \cdots w_{m}
$$

where each $w_{k}$, for $j \leq k \leq m$, corresponds to a path in $C_{k}$ or $w_{k}=1$, the empty word, if $w_{k}$ does not pass through $C_{k}$. Note that since the path corresponding to $w$ originates in $C_{j}$, we must have $w_{j} \neq 1$. We write $v$ in a similar way: if the path corresponding to $v$ originates in $C_{i}$, then

$$
v=v_{i} v_{i+1} \cdots v_{m}
$$

where each $v_{l}$, for $i \leq l \leq m$, corresponds to a path in $C_{l}$ or $v_{l}=1$. Again note that $v_{i} \neq 1$. Since $v<w$, we must have $i \leq j$. We must consider case by case how $v_{i}$ and $w_{j}$ relate given that $v<_{0} w$. If we have $v_{i}=w_{j}$, we then move on to 
compare $v_{i+1}$ and $w_{j+1}$ (hence our use of induction). We proceed by induction on $m-j$.

If $m-j=0$, that is, $j=m$, then $w=w_{m}$. Write $u=u_{1} \cdots u_{m}$ where each $u_{p}$, for $1 \leq p \leq m$ corresponds to a path in $C_{p}$ or $u_{p}=1$.

If $i<j$, observe that

$$
\begin{aligned}
\overline{u v} & =\overline{\left(u_{1} \cdots u_{m}\right)\left(v_{i} \cdots v_{m}\right)} \\
& =\overline{u_{1} \cdots u_{i} v_{i} u_{i+1} v_{i+1} \cdots u_{m} v_{m}}
\end{aligned}
$$

by Lemma 6.9 ; and

$$
u_{1} \cdots u_{i} v_{i} u_{i+1} v_{i+1} \cdots u_{m} v_{m} \in N
$$

by repeated applications of Remark 6.4 . So

$$
\overline{u v}=u_{1} \cdots u_{i} v_{i} u_{i+1} v_{i+1} \cdots u_{m} v_{m} \in N
$$

Similarly, we get

$$
\begin{aligned}
\overline{u w} & =\overline{\left(u_{1} \cdots u_{m}\right) w_{m}} \\
& =u_{1} \cdots u_{i} u_{i+1} \cdots u_{m} w_{m} \in N .
\end{aligned}
$$

We know that $v_{i} \neq 1$ and $v_{i}$ corresponds to a path in a cycle $C_{i}$. If for some $p, i+1 \leq p \leq m$, we have $u_{p} \neq 1$, then $u_{p}$ corresponds to a path in a cycle $C_{p}$ where $p>i$. So if $v_{i}=y v_{i}^{\prime}$, where $y \in\left\{x_{1}, x_{2}, \ldots, x_{n}\right\}$, and $u_{p}=z u_{p}^{\prime}$, where $z \in\left\{x_{1}, x_{2}, \ldots x_{n}\right\}$, then we must have $y<_{0} z$. If $u_{p}=1$ for all $p, i+1 \leq p \leq m$, we know that $w_{m} \neq 1$; so we may write $w_{m}=a w_{m}^{\prime}$, where $a \in\left\{x_{1}, x_{2}, \ldots, x_{n}\right\}$. Again we must have $y<_{0} a$. So

$$
\overline{u v}=u_{1} \ldots u_{i} v_{i} u_{i+1} v_{i+1} \cdots u_{m} w_{m}<_{0} u_{1} \ldots u_{i} \ldots u_{m} w_{m}=\overline{u w} .
$$

That is, we have $u v<u w$.

If $i=j$, then $v=v_{m}$; and we must have $v_{m}<_{0} w_{m}$. So $\overline{u v}=u_{1} \cdots u_{m} v_{m} \in N$, and $\overline{u w}=u_{1} \cdots u_{m} w_{m} \in N$. Since

$$
u_{1} \cdots u_{m} v_{m}<_{0} u_{1} \cdots u_{m} w_{m}
$$

we have $u v<u w$.

Now suppose $m-j>0$; that is, $j<m$. So we may write $w=w_{j} \cdots w_{m}$, where each $w_{k}$, for $j \leq k \leq m$, corresponds to a path in the cycle $C_{k}$ or $w_{k}=1$. Since we assume that the path in $\Gamma(A)$ corresponding to $w$ originates in $C_{j}$, we know that $w_{j} \neq 1$. Again, we write $v$ as $v=v_{i} \cdots v_{m}$, where each $v_{l}$, for $i \leq l \leq m$, corresponds to a path in $C_{l}$ or $v_{l}=1$. Since we assume that the path in $\Gamma(A)$ corresponding to $v$ originates in $C_{i}$, we have that $v_{i} \neq 1$. Since $v<w$, we must have $i \leq j$.

If $i<j$, we have

$$
\begin{aligned}
\overline{u v} & =\overline{\left(u_{1} \cdots u_{m}\right)\left(v_{i} \cdots v_{m}\right)} \\
& =u_{1} \cdots u_{i} v_{i} u_{i+1} v_{i+1} \cdots u_{m} v_{m} \in N
\end{aligned}
$$

and

$$
\begin{aligned}
\overline{u w} & =\overline{\left(u_{1} \cdots u_{m}\right)\left(w_{j} \cdots w_{m}\right)} \\
& =u_{1} \cdots u_{i} \cdots u_{j} w_{j} u_{j+1} w_{j+1} \cdots u_{m} w_{m} \in N .
\end{aligned}
$$


If $u_{p} \neq 1$ for some $p, i+1 \leq p \leq j$, then we may write $v_{i}=y v_{i}^{\prime}$, where $y \in$ $\left\{x_{1}, \ldots, x_{n}\right\}$, and $u_{p}=z u_{p}^{\prime}$, where $z \in\left\{x_{1}, \ldots, x_{n}\right\}$. Since $p>i$, we must have $y<{ }_{0} z$. So

$$
u_{1} \cdots u_{i} v_{i} u_{i+1} v_{i+1} \cdots u_{m} v_{m}<_{0} u_{1} \cdots u_{i} u_{i+1} \ldots u_{j} w_{j} \cdots u_{m} w_{m}
$$

that is, $u v<u w$.

If $u_{p}=1$ for all $p, i+1 \leq p \leq j$, then since we know that $w_{j} \neq 1$, we may write $w_{j}=a w_{j}^{\prime}$, where $a \in\left\{x_{1}, \ldots x_{n}\right\}$. Since $j>i$, we must have $y<_{0} a$. So again we have

$$
u_{1} \cdots u_{i} v_{i} u_{i+1} v_{i+1} \cdots u_{m} v_{m}<_{0} u_{1} \cdots u_{i} u_{i+1} \cdots u_{j} w_{j} u_{j+1} w_{j+1} \cdots u_{m} w_{m}
$$

that is, $u v<u w$.

If $i=j$, then $v=v_{j} \cdots v_{m}$ and $w=w_{j} \cdots w_{m}$, where each $v_{k}, w_{k}$ corresponds to a path in a cycle $C_{k}$ in $\Gamma(A)$, or $v_{k}$ or $w_{k}$ may equal 1 (the empty word). We have

$$
\begin{aligned}
\overline{u v} & =\overline{\left(u_{1} \cdots u_{m}\right)\left(v_{j} \cdots v_{m}\right)} \\
& =u_{1} \cdots u_{j} v_{j} u_{j+1} v_{j+1} \cdots u_{m} v_{m} \in N,
\end{aligned}
$$

and

$$
\begin{aligned}
\overline{u w} & =\overline{\left(u_{1} \cdots u_{m}\right)\left(w_{j} \cdots w_{m}\right)} \\
& =u_{1} \cdots u_{j} w_{j} u_{j+1} w_{j+1} \cdots u_{m} w_{m} \in N .
\end{aligned}
$$

From the assumption that the paths corresponding to $v$ and $w$ both originate in $C_{j}$, we know that $v_{j} \neq 1$ and $w_{j} \neq 1$. So we may write $v_{j}=y_{1} \cdots y_{s}$ and $w_{j}=z_{1} \cdots z_{t}$, where $y_{q}, z_{q^{\prime}} \in\left\{x_{1}, \ldots, x_{n}\right\}$ for each $q, 1 \leq q \leq s$, and for each $q^{\prime}, 1 \leq q^{\prime} \leq t$.

Suppose $s>t$. If there is a $q_{0}, 1 \leq q_{0} \leq t$, such that

$$
y_{1}=z_{1}, \ldots, y_{q_{0}-1}=z_{q_{0}-1} \text {, and } y_{q_{0}}<_{0} z_{q_{0}},
$$

then

$$
u_{1} \cdots u_{j} v_{j} u_{j+1} v_{j+1} \cdots u_{m} v_{m}<_{0} u_{1} \cdots u_{j} w_{j} u_{j+1} w_{j+1} \cdots u_{m} w_{m}
$$

that is, $u v<u w$. But if $y_{1}=z_{1}, \ldots, y_{t}=z_{t}$, then $w_{k} \neq 1$ for some $k, j<k \leq m$. Pick the smallest such $k$ and write $w_{k}=a w_{k}^{\prime}$, where $a \in\left\{x_{1}, \ldots x_{n}\right\}$. Then the first $t+1$ letters of $w$ are $y_{1} \cdots y_{t} a$, and as $k>j$, we must have $y_{t+1}<_{0} a$. Thus,

$$
u_{1} \cdots u_{j} v_{j} u_{j+1} v_{j+1} \cdots u_{m} v_{m}<_{0} u_{1} \cdots u_{j} w_{j} u_{j+1} w_{j+1} \cdots u_{m} w_{m}
$$

that is, $u v<u w$.

Now suppose that $s \leq t$. There are two possibilities:

(1) either there is some $q_{0}, 1 \leq q_{0} \leq s$ such that

$$
y_{1}=z_{1}, \ldots, y_{q_{0}-1}=z_{q_{0}-1} \text { and } y_{q_{0}}<_{0} z_{q_{0}},
$$

or

(2) $s=t$ and $y_{q}=z_{q}$ for all $1 \leq q \leq s$, i.e., $v_{j}=w_{j}$.

If (1) is the case, we proceed as we did when we had $s>t$. If we have (2), then let

$$
\begin{aligned}
v^{\prime} & =v_{j+1} \cdots v_{m}, \\
w^{\prime} & =w_{j+1} \cdots w_{m} .
\end{aligned}
$$


Let

$$
\begin{aligned}
u^{\prime} & =\overline{u v_{j}}\left(=\overline{u w_{j}}\right) \\
& =\overline{\left(u_{1} \cdots u_{m}\right) v_{j}} \\
& =u_{1} \cdots u_{j} v_{j} u_{j+1} \cdots u_{m} \in N .
\end{aligned}
$$

Since $v, w \in N$ and $v<w$, we have $v<_{0} w$. Since $v_{j}=w_{j}$, and $v=v_{j} v^{\prime}$ and $w=w_{j} w^{\prime}$, we must have $v^{\prime}<_{0} w^{\prime}$. So $v^{\prime}<w^{\prime}$, and

$$
\begin{aligned}
\overline{u^{\prime} w^{\prime}} & =\overline{\left(u w_{j}\right)\left(w_{j+1} \cdots w_{m}\right)} \\
& =\overline{u\left(w_{j} w_{j+1} \cdots w_{m}\right)} \\
& =\overline{u w} \neq 0 .
\end{aligned}
$$

Thus, by the induction hypothesis, $u^{\prime} v^{\prime}<u^{\prime} w^{\prime}$, or, equivalently, $\overline{u^{\prime} v^{\prime}}<_{0} \overline{u^{\prime} w^{\prime}}$. But we have shown above that $\overline{u^{\prime} w^{\prime}}=\overline{u w}$; a similar argument shows that $\overline{u^{\prime} v^{\prime}}=\overline{u v}$. So, finally, we have that $\overline{u v}<_{0} \overline{u w}$, or $u v<u w$.

The proof of (2), that $\overline{w u} \neq 0$ implies $v u<w u$, is similar.

Remark 6.11. Let $A=K\langle X\rangle / R$ be a pseudopolynomial ring which is almost commutative on cycles, and suppose $f \in A$. Then we may write

$$
f=\sum_{i=1}^{s} \alpha_{i} u_{i}
$$

where $\alpha_{i} \in K \backslash\{0\}$ and $u_{i} \in N$ such that

$$
u_{s}<_{0} u_{s-1}<_{0} \cdots<_{0} u_{1} .
$$

So $\operatorname{LM}(f)=u_{1}$. Now let $a \in N$ such that $\overline{a u_{1}} \neq 0$. Then $\overline{a f}=\overline{a u_{1}}$ since $u_{1}>_{0} u_{i}$ for $1<i \leq s$ implies $\overline{a u_{1}}>_{0} \overline{a u_{i}}$. We will need this fact in the proof of Theorem 6.15 .

Recall that $T \subset\langle X\rangle$ denotes the set of ordered monomials, i.e., words of the form $x_{1}^{e_{1}} x_{2}^{e_{2}} \cdots x_{n}^{e_{n}}$. The following lemma was proved by Dickson $\mathrm{D}$ ] in the context of commutative polynomial rings.

Dickson's Lemma. For every infinite sequence of ordered monomials $\left\{t_{i} \in T\right.$ : $i=1,2, \ldots\}$, there is an $M$ such that for any $k>M$, there exists $j \leq M$ such that $t_{j}$ divides $t_{k}$.

Dickson's lemma carries over to algebras $A=K\langle X\rangle / R$ where $N(R) \subseteq T$ and the relations in the algebra are such that it makes sense to say that $u=x_{1}^{p_{1}} \cdots x_{n}^{p_{n}}$ divides $v=x_{1}^{q_{1}} \cdots x_{n}^{q_{n}}$ if $p_{i} \leq q_{i}$ for $1 \leq i \leq n$. (That is, there is $a \in N$ such that $\overline{a u}=v$.) Algebras of solvable type fall into this class [KRW], and so do s.f.p. algebras which are strictly ordered and almost commutative, as shown in GI1. But for many pseudopolynomial rings, it is not true that all normal monomials are also ordered monomials; see Examples 6.2, 6.3, and 6.7. So we give a new definition of "divides":

Definition 6.12. Let $u, v \in N$ be such that $u=u_{1} u_{2} \cdots u_{m}$ and $v=v_{1} v_{2} \cdots v_{m}$ where $u_{i}, v_{i}$ correspond to paths in a cycle $C_{i}$ in $\Gamma(A)$ for $1 \leq i \leq m$. If for each $i$, $1 \leq i \leq m$, the paths corresponding to $u_{i}$ and $v_{i}$ begin at the same vertex $y_{i}$ and end at the same vertex $z_{i}$ and $\operatorname{deg}\left(u_{i}\right) \leq \operatorname{deg}\left(v_{i}\right)$, then we say that $u$ divides $v$. 
Example 6.13. We look at the algebra of Example 6.7 again: $A=K\langle x, y, z\rangle /(G)$ where

$$
G=\left\{z^{2}, y^{2}, y x-x y, z x-x z\right\} .
$$

We know that $\operatorname{LM}(G)$ is $\left\{z^{2}, y^{2}, z x, y x\right\}$, and $\Gamma(A)$ is

$$
\mathrm{C}_{x} \longrightarrow y
$$

with cycles $C_{1}=(x \rightarrow x)$ and $C_{2}=(y \rightarrow z \rightarrow y)$. The idea of the definition of "divides" is to split the words in $N$ into (in this case) nine different piles according to how many cycles the corresponding paths in $\Gamma$ pass through, and where the paths enter and exit the cycles. For $A$, we have monomials which correspond to a path in a single cycle: $x^{i},(y z)^{i},(y z)^{i} y,(z y)^{i},(z y)^{i} z, i>0$, and monomials which correspond to a path through both cycles: $x^{i}(y z)^{j}, x^{i}(y z)^{j} y, x^{i}(z y)^{j}, x^{i}(z y)^{j} z$, $i, j>0$. Say we have two monomials in the same pile, e.g., $x^{i}(y z)^{j} z$ and $x^{k}(y z)^{l} z$ for some $i, j, k, l>0$. Then if $i \leq k$ and $j \leq l$, we wish to say that $x^{i}(y z)^{j} z$ divides $x^{k}(y z)^{l} z$ since (using the relations in $R$ )

$$
\overline{\left(x^{k-i}(y z)^{l-j}\right)\left(x^{i}(y z)^{j} z\right)}=x^{k}(y z)^{l} z .
$$

Using this new definition of "divides," we get a generalized version of Dickson's lemma:

Lemma 6.14. Let $S=\left\{u_{k} \in N: k=1,2, \ldots\right\}$ be an infinite sequence of monomials such that each $u_{k}$ may be written as

$$
u_{k}=u_{k 1} u_{k 2} \cdots u_{k m}
$$

where each $u_{k i}$ corresponds to a path in a cycle $C_{i}$ in $\Gamma(A)$ for $1 \leq i \leq m$, and for a given $i$, all $u_{k i}($ for $k=1,2, \ldots)$ begin at the same vertex $y_{i}$ and end at the same vertex $z_{i}$ in $C_{i}$. Then there exists an $M$ such that for any $k>M$ there is $j \leq M$ such that $u_{j}$ divides $u_{k}$.

Proof. We proceed by induction on $m$.

If $m=1$, then $u_{k}=u_{k 1}$ for each $k$; i.e., each $u_{k}$ corresponds to a path in a cycle $C_{1}=\left(x_{i_{1}} \rightarrow x_{i_{2}} \rightarrow \cdots \rightarrow x_{i_{t}} \rightarrow x_{i_{1}}\right)$ and each begins at the same vertex $x_{i_{1}}$ and ends at the same vertex $x_{i_{e}}$, where $1 \leq e \leq t$. So each $u_{k}$ is of the form $u_{k}=\gamma^{i} w$, for some $i \geq 0$, where $\gamma$ corresponds to the path $x_{i_{1}} \rightarrow x_{i_{2}} \rightarrow \cdots \rightarrow x_{i_{t}}$ and $w$ corresponds to the path $x_{i_{1}} \rightarrow x_{i_{2}} \rightarrow \cdots \rightarrow x_{i_{e}}$ (or if $x_{i_{1}}=x_{i_{e}}$, then $w=1$ ).

Let $p=\min \left\{i: \gamma^{i} w=u_{k}\right.$ for some $\left.k\right\}$. Then there is an $M$ such that $u_{M}=\gamma^{p} w$. Clearly $u_{M}$ divides $u_{k}$ for any $k \geq M$.

Now let $m>1$. Each $u_{k}$ may be written as

$$
u_{k}=u_{k 1} u_{k 2} \cdots u_{k m} .
$$

Let

$$
v_{k}=u_{k 1} u_{k 2} \cdots u_{k(m-1)},
$$

and look at the sequence $T=\left\{v_{k} \in N: k=1,2, \ldots\right\}$. By the induction hypothesis, we can find $k_{0}$ such that for any $k>k_{0}$, there is $j \leq k_{0}$ such that $v_{j}$ divides $v_{k}$.

For each $k, 1 \leq k \leq k_{0}$, there is a $u_{k m}$ such that $v_{k} u_{k m}=u_{k}$. If $C_{m}=$ $\left(x_{i_{1}} \rightarrow x_{i_{2}} \rightarrow \cdots \rightarrow x_{i_{t}}\right)$, we note that each $u_{k m}$ is of the form $u_{k m}=\gamma^{i} w$ for some $i \geq 0$, where $\gamma$ corresponds to the path $x_{i_{1}} \rightarrow x_{i_{2}} \rightarrow \cdots \rightarrow x_{i_{t}}$ and $w$ corresponds to the path $x_{i_{1}} \rightarrow x_{i_{2}} \rightarrow \cdots \rightarrow x_{i_{e}}$ if $2 \leq e \leq t$. If $e=1$, then $w=1$. So for each $k, 1 \leq k \leq k_{0}$, we have $u_{k}=v_{k} \gamma^{i(k)} w$ for some $i(k) \geq 0$. 
Let $L=\max \left\{i(k): 1 \leq k \leq k_{0}\right\}$.

Consider the following subsequences of $T$ :

$$
\begin{gathered}
V_{0}=\left\{v_{k_{j}} \in T: u_{k_{j}}=v_{k_{j}} w \in S, j=1,2, \ldots\right\} \\
V_{1}=\left\{v_{k_{j}} \in T: u_{k_{j}}=v_{k_{j}} \gamma w \in S, j=1,2, \ldots\right\} \\
\quad \vdots \\
V_{i}=\left\{v_{k_{j}} \in T: u_{k_{j}}=v_{k_{j}} \gamma^{i} w \in S, j=1,2, \ldots\right\} \\
\vdots \\
V_{L-1}=\left\{v_{k_{j}} \in T: u_{k_{j}}=v_{k_{j}} \gamma^{L-1} w \in S, j=1,2, \ldots\right\} .
\end{gathered}
$$

By the induction hypothesis, there exist $j_{0}, j_{1}, \ldots, j_{L-1}$ such that for any $i, 0 \leq$ $i \leq L-1$, for any $j>j_{i}$, for $v_{k_{j}} \in V_{i}$, there is a $p \leq j_{i}$ such that $v_{k_{p}}$ divides $v_{k_{j}}$.

Let $M=\max \left\{k_{0}, k_{j_{0}}, \ldots, k_{j_{(L-1)}}\right\}$.

We now show that for any $k>M$, there is a $j \leq M$ such that $u_{j}$ divides $u_{k}$. Write $u_{k}$ as $u_{k}=v_{k} \gamma^{i} w$. If $i \geq L$, then there is a $j \leq k_{0} \leq M$ such that $v_{j}$ divides $v_{k}$. Then $u_{j}=v_{j} \gamma^{i(j)} w$ divides $u_{k}$ since $i(j) \leq L \leq i$.

If $i<L$, then $v_{k} \in V_{i}$. So there is a $j \leq j_{i} \leq M$ such that $v_{k_{j}}$ divides $v_{k}$. So $u_{k_{j}}=v_{k_{j}} \gamma^{i} w$ divides $v_{k} \gamma^{i} w=u_{k}$.

We now come to the main result.

Theorem 6.15. Suppose $A$ is a pseudopolynomial ring. Then, if $A$ is almost commutative on cycles, $A$ is Noetherian.

Proof. Let $J$ be a left ideal in $A$. The idea is that we wish to look at the ideal generated by $\operatorname{LM}(J)$, which is a monomial ideal, and find a finite generating set using Lemma 6.14. To use the lemma, we will need to use the graph $\Gamma(A)$ to split the monomials of $\operatorname{LM}(J)$ into several different piles using the definition of "divides" introduced above. Then we use Lemma 6.14 on each pile separately, finally gathering the generators we find in this way into one big pile which generates all of $(\operatorname{LM}(J))$. We find a set of polynomials in $J$ which has the generators of $(\operatorname{LM}(J))$ as leading monomials. We then show that these polynomials generate $J$.

We may assume that all elements of $J$ are in normal form; i.e., $J \subseteq \operatorname{Span} N$. So

$$
\operatorname{LM}(J)=\{\bar{g}: g \in J\} \subseteq N ;
$$

that is, each $u \in \operatorname{LM}(J)$ corresponds to a path in $\Gamma(A)$. Clearly $\operatorname{LM}(J)$ is countable. Let the sequence

$$
U=\left\{u_{1}, u_{2}, u_{3}, \ldots\right\}
$$

be an enumeration of $\operatorname{LM}(J)$ where $u_{i}<u_{j}$ for $1 \leq i<j$.

Since $\Gamma(A)$ is a finite graph, we may partition $U$ into a finite number of subsequences $U_{1}, U_{2}, \ldots, U_{r}$ such that for any $v, w \in U_{i}, 1 \leq i \leq r$, we may write

$$
v=v_{1} v_{2} \cdots v_{m} \text { and } w=w_{1} w_{2} \cdots w_{m},
$$

where $v_{j}, w_{j}$ correspond to paths in a cycle $C_{j}$ in $\Gamma(A)$ for $1 \leq j \leq m$ and for each $j, 1 \leq j \leq m$, the paths corresponding to $v_{j}$ and $w_{j}$ begin at the same vertex $y_{j}$ 
and end at the same vertex $z_{j}$. Note that

$$
U=\bigcup_{i=1}^{r} U_{i} .
$$

For each $i, 1 \leq i \leq r$, write

$$
U_{i}=\left\{u_{i_{k}}: k=1,2, \ldots\right\} .
$$

By Lemma 6.14, there exists $k(i)$ such that for any $k>k(i)$, there is a $j \leq k(i)$ such that $u_{i_{j}}$ divides $u_{i_{k}}$. Since $u_{i_{k(i)}} \in U_{i}$, we have $u_{i_{k(i)}}=u_{p(i)} \in U$ for some $p(i)$. Let $M=\max \{p(i): 1 \leq i \leq r\}$. Then for any $k>M$, there is a $j \leq M$ such that $u_{j}$ divides $u_{k}$. Clearly, there exist elements $f_{1}, f_{2}, \ldots, f_{M}$ of $J$ such that

$$
f_{i}=u_{i}-\varphi_{i}
$$

where $\varphi_{i} \in \operatorname{Span} N$ and (if $\varphi_{i} \neq 0$ ) $\operatorname{LM}\left(\varphi_{i}\right)<u_{i}, 1 \leq i \leq M$. Let $F=$ $\left\{f_{1}, f_{2}, \ldots, f_{M}\right\}$.

Let $g \in J$, and let $u=\bar{g}$. Since $u \in \operatorname{LM}(J)$, by choice of $M$, there exists $j \leq M$ such that $u_{j}$ divides $u$; i.e., $u=v_{1} \cdots v_{m}$ and $u_{j}=v_{j 1} \cdots v_{j m}$ where $v_{i}, v_{j i}$ correspond to paths in $C_{i}$ in $\Gamma(A)$ for $1 \leq i \leq m$. For each $i$, the paths corresponding to $v_{i}$ and $v_{j i}$ begin at the same vertex $y_{i}$ and end at the same vertex $z_{i}$, and $\operatorname{deg}\left(v_{j i}\right) \leq \operatorname{deg}\left(v_{i}\right)$. That is, for each $i$, we have

$$
v_{i}=\gamma_{i}^{p(i)} w_{i} \text { and } v_{j i}=\gamma_{i}^{q(i)} w_{i}
$$

where $\gamma_{i}$ corresponds to the path once around the cycle $C_{i}=\left(x_{i_{1}} \rightarrow x_{i_{2}} \rightarrow \cdots \rightarrow\right.$ $\left.x_{i_{t(i)}} \rightarrow x_{i_{1}}\right)$ and $w_{i}$ corresponds to the path $x_{i_{1}} \rightarrow \cdots \rightarrow x_{i_{e(i)}}$, where $2 \leq e(i) \leq$ $t(i)$ (or if $y_{i}=z_{i}$, then $w_{i}=1$ ). Since $\operatorname{deg}\left(v_{j i}\right) \leq \operatorname{deg}\left(v_{i}\right)$, we have $q(i) \leq p(i)$. Let $s(i)=p(i)-q(i)$, and let $a=\gamma_{1}^{s(1)} \ldots \gamma_{m}^{s(m)}$. (If $s(i)=0$ for some $i$, then we let $\gamma_{i}^{s(i)}=1$.) The monomial $a \in N$ since condition (3) on pseudopolynomial rings tells us that

$$
\gamma_{i} \gamma_{i+1} \in N \text { for each } 1 \leq i \leq n-1 \text {; }
$$

so we may apply Remark 6.4. Then, since $A$ is almost commutative on cycles, we have

$$
\begin{aligned}
\overline{a u_{j}} & =\overline{\left(\gamma_{1}^{s(1)} \cdots \gamma_{m}^{s(m)}\right)\left(\gamma_{1}^{q(1)} w_{1} \cdots \gamma_{m}^{q(m)} w_{m}\right)} \\
& =\gamma_{1}^{s(1)} \gamma_{1}^{q(1)} w_{1} \cdots \gamma_{m}^{s(m)} \gamma_{m}^{q(m)} w_{m} \\
& =u .
\end{aligned}
$$

We now show that $F$ generates $J$ as a left ideal. Suppose $g \in J$ and $\operatorname{LM}(g)=u$. Then there is a $j_{1} \leq M$ such that $u_{j_{1}}$ divides $u$. Let $a_{1} \in N$ be such that $\overline{a_{1} u_{j_{1}}}=u$. Then by Remark 6.11 , we know that $\overline{a_{1} f_{j_{1}}}=u$. For notational convenience, let $g=g_{0}$. Then let

$$
g_{1}=g_{0}-\mathrm{LC}\left(g_{0}\right) \operatorname{Nor}\left(a_{1} f_{j_{1}}\right)
$$

and let

$$
h_{1}=\mathrm{LC}\left(g_{0}\right) \operatorname{Nor}\left(a_{1} f_{j_{1}}\right) .
$$


Note that $\operatorname{LM}\left(g_{1}\right)<\operatorname{LM}(g)$ and $g=g_{1}+h_{1}$. Now for $k \geq 1$, if $\operatorname{LM}\left(g_{k}\right) \neq 0$, then there exists $j_{k} \leq M$ such that $u_{j_{k}}$ divides $\operatorname{LM}\left(g_{k}\right)$. So there is an $f_{j_{k}} \in F$ such that $\operatorname{LT}\left(f_{j_{k}}\right)=u_{j_{k}}$. Let $a_{k} \in N$ be such that $\overline{a_{k} u_{j_{k}}}=\operatorname{LM}\left(g_{k}\right)$. Then let

$$
g_{k+1}=g_{k}-\mathrm{LC}\left(g_{k}\right) \operatorname{Nor}\left(a_{k} f_{j_{k}}\right)
$$

and

$$
h_{k+1}=h_{k}+\operatorname{LC}\left(g_{k}\right) \operatorname{Nor}\left(a_{k} f_{j_{k}}\right) .
$$

For each $k \geq 1$, we have $\operatorname{LM}\left(g_{k+1}\right)<\operatorname{LM}\left(g_{k}\right)$ and $g=g_{k}+h_{k}$. Since we have a well-founded term ordering, we must have $g_{s}=0$ for some $s$. Then

$$
g=h_{s}=\sum_{l=1}^{s} \mathrm{LC}\left(g_{l-1}\right) \operatorname{Nor}\left(a_{l} f_{j_{l}}\right) .
$$

That is, $F$ is a left basis for $J$.

The proof that $A$ is right Noetherian is similar.

There is a lot of redundancy built into our new notion of "divides" and thus in the proofs of Lemma 6.14 and Theorem 6.15. Consider again the algebra of Examples 6.7 and 6.13: $A=K\langle x, y, z\rangle /(G)$ where

$$
G=\left\{z^{2}, y^{2}, y x-x y, z x-x z\right\} .
$$

Suppose $I$ is a left ideal of $A$. To find a left basis for $I$, we first find a left basis for $(\mathrm{LM}(I))$, which is a monomial ideal in $A$. Considering monomials of the form $(y z)^{j}$ and $x^{i}(y z)^{j}$ separately is somewhat inefficient; after all, if we have a monomial $(y z)^{j}$, we can left multiply by $x^{i}$ to obtain $x^{i}(y z)^{j}$. We could minimize this redundancy by introducing a notion of "left divides" (or "divides with respect to left multiplication"), but this approach unnecessarily complicates the proofs of Lemma 6.14 and Theorem 6.15. This tactic will be useful in Example 6.16, however, since we will need two separate arguments to show that the algebra considered there is both right and left Noetherian.

The converse to Theorem 6.15 is false in general. For a skew polynomial ring $A=K\left\langle x_{1}, x_{2}, \ldots, x_{n}\right\rangle / R$, the cycles in $\Gamma(A)$ are all of the form $\left(x_{i} \rightarrow x_{i}\right)$ for $1 \leq i \leq n$; so a skew polynomial ring is almost commmutative on cycles if and only if it is almost commutative. But Theorem 5.8 implies that there are many examples of Noetherian binomial skew polynomial rings that are not almost commutative. For instance, suppose $A=K\left\langle x_{1}, x_{2}, x_{3}\right\rangle /(G)$ where

$$
G=\left\{x_{3} x_{2}-x_{1} x_{3}, x_{3} x_{1}-x_{2} x_{3}, x_{2} x_{1}-x_{1} x_{2}\right\} .
$$

One can easily check that $G$ is a Gröbner basis; so $A$ is indeed a binomial skew polynomial ring. The set $\left\{x_{i^{\prime}} x_{j^{\prime}}: 1 \leq i<j \leq n\right\}$ of Theorem 5.8 is $\left\{x_{1} x_{3}, x_{2} x_{3}, x_{1} x_{2}\right\}$ which does indeed have $\left(\begin{array}{l}3 \\ 2\end{array}\right)=3$ elements. So $A$ is Noetherian, but since $x_{3} x_{2} \nsim$ $x_{2} x_{3}$, it is not almost commutative.

We also give an example of a pseudopolynomial ring which is not a skew polynomial ring and is not almost commutative on cycles, but is Noetherian.

Example 6.16. Consider again $A=K\langle x, y, z\rangle / R$ where

$$
R=\left(z^{2}, y^{2}, z x-x y, y x-x z\right),
$$


the algebra of Examples 6.7 and 6.13. The Ufnarovskii graph $\Gamma(A)$ is

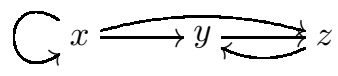

Clearly, the algebra $A$ is not almost commutative on cycles since $y x \nsim x y$, but $A$ is Noetherian.

To show that $A$ is left Noetherian, we first show that all monomial ideals of $A$ are finitely generated. So let $I$ be a monomial left ideal in $A$, and let $G=$ $\left\{m_{p} \in N: p \in P\right\}$ be a (possibly infinite) set of generators for $I$; so $I=(G)$. Let

$$
\begin{aligned}
J & =\left(x^{i}: i \geq 0, x^{i} \in I\right), \\
Y_{0} & =\left(x^{i}: i \geq 0, x^{i}(y z)^{j} \in I \text { for some } j>0\right), \\
Y_{1} & =\left(x^{i}: i \geq 0, x^{i}(y z)^{j} y \in I \text { for some } j \geq 0\right), \\
Z_{0} & =\left(x^{i}: i \geq 0, x^{i}(z y)^{j} \in I \text { for some } j>0\right), \\
Z_{1} & =\left(x^{i}: i \geq 0, x^{i}(z y)^{j} z \in I \text { for some } j \geq 0\right) .
\end{aligned}
$$

(Notice that if there are any monomials in $I$ of the form $(y z)^{j}$, then $1 \in Y_{0}$. Similarly for $Y_{1}, Z_{0}$, and $Z_{1}$.) Then $J\left(\right.$ if $J \neq \emptyset$ ) is generated by some $x^{\alpha}$. Similarly, $Y_{0}, Y_{1}, Z_{0}, Z_{1}$ are generated by $x^{\beta}, x^{\gamma}, x^{\delta}, x^{\epsilon}$, respectively. So there is an $m>0$ such that $x^{\beta}(y z)^{m} \in I$. There could be monomials in $I$ of the form $x^{i}(y z)^{j}$ where $j<m$ (and of course $i>\beta$ by the way $\beta$ was chosen); so to avoid missing any of these monomials we define the following ideals. Let

$$
\begin{gathered}
S_{1}=\left(x^{i}: i \geq 0, x^{i}(y z) \in I\right) \\
S_{2}=\left(x^{i}: i \geq 0, x^{i}(y z)^{2} \in I\right) \\
\vdots \\
S_{k}=\left(x^{i}: i \geq 0, x^{i}(y z)^{k} \in I\right) \\
\vdots \\
S_{m-1}=\left(x^{i}: i \geq 0, x^{i}(y z)^{m-1} \in I\right) .
\end{gathered}
$$

Each nonempty $S_{k}$ is generated by some $x^{\beta(k)}$ for $1 \leq k \leq m-1$. (Note: Throughout this proof, the letter $k$ will be used as a generic index.)

We now proceed in the same way with the generators of $Y_{0}, Y_{1}, Z_{0}$, and $Z_{1}$. There is an $n \geq 0$ such that $x^{\gamma}(y z)^{n} y \in I$. Let

$$
\begin{gathered}
T_{0}=\left(x^{i}: i \geq 0, x^{i} y \in I\right) \\
T_{1}=\left(x^{i}: i \geq 0, x^{i}(y z) y \in I\right) \\
\vdots \\
T_{k}=\left(x^{i}: i \geq 0, x^{i}(y z)^{k} y \in I\right) \\
\vdots \\
T_{n-1}=\left(x^{i}: i \geq 0, x^{i}(y z)^{n-1} y \in I\right) .
\end{gathered}
$$

Each nonempty $T_{k}$ is generated by some $x^{\gamma(k)}$ for $1 \leq k \leq n-1$. 
There is an $l>0$ such that $x^{\delta}(z y)^{l} \in I$. Let

$$
\begin{gathered}
U_{1}=\left(x^{i}: i \geq 0, x^{i}(z y) \in I\right) \\
\vdots \\
U_{k}=\left(x^{i}: i \geq 0, x^{i}(z y)^{k} \in I\right) \\
\vdots \\
U_{l-1}=\left(x^{i}: i \geq 0, x^{i}(z y)^{l-1} \in I\right) .
\end{gathered}
$$

Each nonempty $U_{k}$ is generated by some $x^{\delta(k)}$ for $1 \leq k \leq l-1$.

There is a $q \geq 0$ such that $x^{\epsilon}(z y)^{q} z \in I$. Let

$$
\begin{gathered}
V_{0}=\left(x^{i}: i \geq 0, x^{i} z \in I\right) \\
V_{1}=\left(x^{i}: i \geq 0, x^{i}(z y) z \in I\right) \\
\vdots \\
V_{k}=\left(x^{i}: i \geq 0, x^{i}(z y)^{k} z \in I\right) \\
\vdots \\
V_{q-1}=\left(x^{i}: i \geq 0, x^{i}(z y)^{q-1} z \in I\right) .
\end{gathered}
$$

Each nonempty $V_{k}$ is generated by some $x^{\epsilon(k)}$ for $1 \leq k \leq q-1$.

Finally, we put all the generators we have found into one big pile. Let

$$
\begin{aligned}
G^{\prime}=\{ & x^{\alpha}, x^{\beta}(y z)^{m}, x^{\beta(k)}(y z)^{k} \text { for } 1 \leq k \leq m-1, \\
& x^{\gamma}(y z)^{n} y, x^{\gamma(k)}(y z)^{k} y \text { for } 1 \leq k \leq n-1, \\
& x^{\delta}(z y)^{l}, x^{\delta(k)}(z y)^{k} \text { for } 1 \leq k \leq l-1, \\
& \left.x^{\epsilon}(z y)^{q} z, x^{\epsilon(k)}(z y)^{k} z \text { for } 1 \leq k \leq q-1\right\} .
\end{aligned}
$$

We show that $G^{\prime}$ generates $I$ as a left ideal.

Let $u \in G$.

Case 1. Suppose $u=x^{i}$ for some $i \geq 0$. Then $u \in J$; so $i \geq \alpha$ and $x^{i-\alpha}\left(x^{\alpha}\right)=x^{i}$. Note that $x^{\alpha} \in G^{\prime}$.

Case 2. Suppose $u=x^{i}(y z)^{j}$ for some $i \geq 0, j>0$.

Subcase (a). Suppose $j<m$. Then $x^{i} \in S_{j}$; so $i \geq \beta(j)$ and

$$
x^{i-\beta(j)}\left(x^{\beta(j)}(y z)^{j}\right)=x^{i}(y x)^{j} .
$$

Note that $x^{\beta(j)}(y z)^{j} \in G^{\prime}$.

Subcase (b). Suppose $j \geq m$. Then $x^{i} \in Y_{0}, i \geq \beta$, and $x^{i-\beta}\left(x^{\beta}\right)=x^{i}$. If $\beta$ is even, then

$$
\overline{\left(x^{i-\beta}(y z)^{j-m}\right)\left(x^{\beta}(y z)^{m}\right)}=x^{i}(y z)^{j} .
$$

Actually, for this algebra, we really have

$$
\operatorname{Nor}\left(x^{i-\beta}(y z)^{j-m}\right)\left(x^{\beta}(y z)^{m}\right)=x^{i}(y z)^{j} .
$$

If $\beta$ is odd, then

$$
\overline{\left(x^{i-\beta}(z y)^{j-m}\right)\left(x^{\beta}(y z)^{m}\right)}=x^{i}(y z)^{j} .
$$

The monomial $x^{\beta}(y z)^{m} \in G^{\prime}$. 
Case 3. Suppose $u=x^{i}(y z)^{j} y$ for some $i \geq 0, j \geq 0$.

Subcase (a). Suppose $j<n$. Then $x^{i} \in T_{j}$; so $i \geq \gamma(j)$ and

$$
x^{i-\gamma(j)}\left(x^{\gamma(j)}(y z)^{j} y\right)=x^{i}(y z)^{j} y,
$$

where $x^{\gamma(j)}(y z)^{j} y \in G^{\prime}$.

Subcase (b). Suppose $j \geq n$. Then $x^{i} \in Y_{1}, i \geq \gamma$, and $x^{i-\gamma}\left(x^{\gamma}\right)=x^{i}$. If $\gamma$ is even, then

$$
\overline{\left(x^{i-\gamma}(y z)^{j-n}\right)\left(x^{\gamma}(y z)^{n} y\right)}=x^{i}(y z)^{j} y .
$$

If $\gamma$ is odd, then

$$
\overline{\left(x^{i-\gamma}(z y)^{j-n}\right)\left(x^{\gamma}(y z)^{n} y\right)}=x^{i}(y z)^{j} y .
$$
2 .

Case 4. Suppose $u=x^{i}(z y)^{j}$ for some $i \geq 0, j>0$. This case is similar to Case

Case 5. Suppose $u=x^{i}(z y)^{j} z$ for some $i \geq 0, j \geq 0$. This case is similar to Case 3.

We have shown that any monomial left ideal in $A$ is finitely generated. Now let $I$ be an arbitrary ideal of $A$. The ideal $(\operatorname{LM}(I))$ must be finitely generated, say by $u_{1}, u_{2}, \ldots, u_{s}$. For each $u_{i}$, there is an $f_{i} \in I$ such that $\operatorname{LM}\left(f_{i}\right)=u_{i}$. Let $F=\left\{f_{i}: 1 \leq i \leq s\right\}$. For this algebra, it is easily seen that for $u, v, w \in N$ such that $v<w$ and $\overline{u w} \neq 0$, we have $u v<u w$. Thus, we may use the procedure given in the proof of Theorem 6.15 to show that $F$ generates $I$ as a left ideal.

To see that $A$ is right Noetherian, we will need a somewhat different argument, since it is not true that for $u, v, w \in N$ such that $v<w$ and $\overline{w u} \neq 0$, we always have $v u<w u$. For instance, $y<z$, but

$$
\overline{y x}=x z \nless_{0} \overline{z x}=x y ;
$$

i.e., $y x \nless z x$. However, if we know that $u$ is of the form $x^{i} u^{\prime}$ where $i$ is even and $u^{\prime}$ corresponds to a path in the second cycle, then we may conclude that $v u<w u$. So $y<z$, and

$$
\overline{y x^{2}}=x^{2} y<\overline{z x^{2}}=x^{2} z .
$$

The point is that as long as we multiply by even powers of $x$, we are safe, and being able to multiply by these even powers turns out to be good enough. We again start with a monomial ideal: let $I$ be a monomial right ideal in $A$, and let $G=\left\{m_{p} \in N: p \in P\right\}$ be a (possibly infinite) set of generators for $I$; so $I=(G)$.

Let

$$
Y=\left\{(y z)^{j} y^{\epsilon}: j \geq 0, \epsilon=0 \text { or } 1, x^{i}(y z)^{j} y^{\epsilon} \in I \text { for some } i \geq 0\right\},
$$

and let

$$
Z=\left\{(z y)^{j} z^{\epsilon}: j \geq 0, \epsilon=0 \text { or } 1, x^{i}(y z)^{j} y^{\epsilon} \in I \text { for some } i \geq 0\right\} .
$$

Let $u$ be the element of $Y$ of least degree. Then for any $m \in Y$, there exists $a \in K\langle x, y, z\rangle$ such that $u a=m$. Similarly, let $v$ be the element of $Z$ of least degree.

There is an $\alpha$ such that $x^{\alpha} u \in I$ and a $\beta$ such that $x^{\beta} v \in I$. 
Let

$$
\begin{gathered}
Y_{0}=\left\{(y z)^{j} y^{\epsilon}: j \geq 0, \epsilon=0 \text { or } 1,(y z)^{j} y^{\epsilon} \in I\right\} \\
Y_{1}=\left\{(y z)^{j} y^{\epsilon}: j \geq 0, \epsilon=0 \text { or } 1, x(y z)^{j} y^{\epsilon} \in I\right\} \\
\vdots \\
Y_{k}=\left\{(y z)^{j} y^{\epsilon}: j \geq 0, \epsilon=0 \text { or } 1, x^{k}(y z)^{j} y^{\epsilon} \in I\right\} \\
\vdots \\
Y_{\alpha-1}=\left\{(y z)^{j} y^{\epsilon}: j \geq 0, \epsilon=0 \text { or } 1, x^{\alpha-1}(y x)^{j} y^{\epsilon} \in I\right\} .
\end{gathered}
$$

Let $u_{k}$ be the element of $Y_{k}$ of minimal degree for $0 \leq k \leq \alpha-1$.

Let

$$
\begin{gathered}
Z_{0}=\left\{(z y)^{j} z^{\epsilon}: j \geq 0, \epsilon=0 \text { or } 1,(z y)^{j} z^{\epsilon} \in I\right\} \\
\vdots \\
Z_{k}=\left\{(z y)^{j} z^{\epsilon}: j \geq 0, \epsilon=0 \text { or } 1, x^{k}(z y)^{j} z^{\epsilon} \in I\right\} \\
\vdots \\
Z_{\beta-1}=\left\{(z y)^{j} z^{\epsilon}: j \geq 0, \epsilon=0 \text { or } 1, x^{\beta-1}(z y)^{j} z^{\epsilon} \in I\right\} .
\end{gathered}
$$

Let $v_{k}$ be the element of $Z_{k}$ of minimal degree for $0 \leq k \leq \beta-1$.

Let

$$
E_{y}=\left\{m \in I: m=x^{i}(y z)^{j} y^{\epsilon}, i \geq \alpha, \text { and } i-\alpha \text { is even }\right\}
$$

and let

$$
O_{y}=\left\{m \in I: m=x^{i}(y z)^{j} y^{\epsilon}, i>\alpha \text {, and } i-\alpha \text { is odd }\right\} .
$$

Note that for any two elements $x^{i_{1}}(y z)^{j_{1}} y^{\epsilon_{1}}$ and $x^{i_{2}}(y z)^{j_{2}} y^{\epsilon_{2}}$ of $O_{y}, i_{1}-i_{2}$ is even.

Let

$$
E_{z}=\left\{m \in I: m=x^{i}(z y)^{j} z^{\epsilon}, i \geq \beta, \text { and } i-\beta \text { is even }\right\}
$$

and let

$$
O_{z}=\left\{m \in I: m=x^{i}(z y)^{j} z^{\epsilon}, i>\beta, \text { and } i-\beta \text { is odd }\right\} .
$$

Let

$$
S=\left\{(y z)^{j} y^{\epsilon}: x^{i}(y z)^{j} y^{\epsilon} \in O_{y}\right\}
$$

and let

$$
T=\left\{(z y)^{j} z^{\epsilon}: x^{i}(z y)^{j} x^{\epsilon} \in O_{z}\right\} .
$$


Let $w_{1}$ be the element in $S$ of minimal degree and $w_{2}$ the element in $T$ of minimal degree. Then there exist $\gamma, \delta$ such that $x^{\gamma} w_{1} \in O_{y}$ and $x^{\delta} w_{2} \in O_{z}$. Let

$$
\begin{aligned}
U_{1} & =\left\{(y z)^{j} y^{\epsilon}: x^{\alpha+1}(y z)^{j} y^{\epsilon} \in O_{y}\right\} \\
\vdots & \\
U_{k} & =\left\{(y z)^{j} y^{\epsilon}: x^{\alpha+k}(y z)^{j} y^{\epsilon} \in O_{y}\right\} \\
\vdots & \\
U_{(\gamma-\alpha-2)} & =\left\{(y z)^{j} y^{\epsilon}: x^{\gamma-2}(y z)^{j} y^{\epsilon} \in O_{y}\right\} .
\end{aligned}
$$

Let the element of minimal degree in $U_{k}$ be $s_{k}$ for $1 \leq k \leq \gamma-\alpha-2$.

Let

$$
\begin{array}{r}
V_{1}=\left\{(z y)^{j} z^{\epsilon}: x^{\beta+1}(z y)^{j} z^{\epsilon} \in O_{z}\right\} \\
\vdots \\
V_{k}=\left\{(z y)^{j} z^{\epsilon}: x^{\beta+k}(z y)^{j} z^{\epsilon} \in O_{z}\right\} \\
\vdots \\
V_{(\delta-\beta-2)}=\left\{(z y)^{j} z^{\epsilon}: x^{\delta-2}(z y)^{j} z^{\epsilon} \in O_{z}\right\} .
\end{array}
$$

Let the element of minimal degree in $V_{k}$ be $t_{k}$ for $1 \leq k \leq \delta-\beta-2$.

Now let

$$
\begin{aligned}
G^{\prime}=\{ & x^{\alpha} u, x^{\beta} v, x^{k} u_{k} \text { for } 0 \leq k \leq \alpha-1, \\
& x^{k} v_{k} \text { for } 0 \leq k \leq \beta-1, \\
& x^{\gamma} w_{1}, x^{\delta} w_{2}, \\
& x^{\alpha+k} s_{k} \text { for } 1 \leq k \leq \gamma-\alpha-2, \\
& \left.x^{\beta+k} t_{k} \text { for } 1 \leq k \leq \delta-\beta-2\right\} .
\end{aligned}
$$

We show that $G^{\prime}$ generates $I$ as a right ideal.

Let $m \in G$.

Case 1. Suppose $m=x^{i}(y z)^{j} y^{\epsilon}$ for some $i \geq 0, j \geq 0, \epsilon=0$ or 1 .

Subcase (a). Suppose $i<\alpha$. Then $(y z)^{j} y^{\epsilon} \in Y_{i}$ and there is an $a \in K\langle x, y, z\rangle$ such that $u_{i} a=(y z)^{j} y^{\epsilon}$. So $x^{i} u_{i} \in G^{\prime}$ and $\overline{\left(x^{i} u_{i}\right) a}=x^{i}(y z)^{j} y^{\epsilon}$.

Subcase (b). Suppose $i \geq \alpha$ and $i-\alpha$ is even; i.e., $m \in E_{y}$. Then there is an $a \in K\langle x, y, z\rangle$ such that $u a=(y z)^{j} y^{\epsilon}$. So $\overline{x^{\alpha} u\left(x^{i-\alpha} a\right)}=x^{i}(y z)^{j} y^{\epsilon}$.

Subcase (c). Suppose $i>\alpha$ and $i-\alpha$ is odd; i.e., $m \in O_{y}$. If $\alpha+1 \leq i<\gamma$, then there is an $a \in K\langle x, y, z\rangle$ such that $s_{i} a=(y z)^{j} y^{\epsilon}$. So $\left(x^{i} s_{i}\right) a=x^{i}(y z)^{j} y^{\epsilon}$. If $i \geq \gamma$, then there is an $a \in K\langle x, y, z\rangle$ such that $w_{1} a=(y z)^{j} y^{\epsilon}$. Note that $i-\gamma$ is even. So $\overline{\left(x^{\gamma} w_{1}\right)\left(x^{i-\gamma} a\right)}=x^{i}(y z)^{j} y^{\epsilon}$.

Case 2. Suppose $m=x^{i}(z y)^{j} z^{\epsilon}$. This case is similar to Case 1 .

We have shown that any monomial right ideal of $A$ is finitely generated. Note that we have chosen our generators so that we will always multiply them by even powers of $x$. It is now easy to show that an arbitrary right ideal of $A$ is finitely generated using the procedure given in the proof of Theorem 6.15.

In conclusion, we note that two very natural questions remain unanswered. First, Theorem 6.15 shows that if a pseudopolynomial ring is almost commutative on 
cycles, then it is Noetherian; however, by Example 6.16 we know that a Noetherian pseudopolynomial ring need not be almost commutative on cycles. So what must be true of any Noetherian pseudopolynomial ring? That is, what are some necessary conditions on a pseudopolynomial ring if it is to be Noetherian? This question is answered by Gateva-Ivanova for some binomial skew polynomial rings in GI3] (here cited as Theorem 5.8) and in [GI4.

Second, recall from Definition 5.5 that an algebra $A=K\left\langle x_{1}, \ldots, x_{n}\right\rangle / I$ is strictly ordered if

$$
\operatorname{deg}\left(\overline{x_{i} x_{j}}\right)=2 \text { for } 1 \leq i, j \leq n,
$$

and if for $u, v, w \in N(I)$ such that $v<w$, we have $u v<u w$ and $v u<w u$. In a pseudopolynomial ring $B$ that is almost commutative on cycles, we get similar, though somewhat weaker, conditions. That is, for $B$ we have

(1) if $x_{i}, x_{j}$ are vertices in distinct cycles of the Ufnarovskii graph, then $\overline{x_{i} x_{j}} \neq 0$ (from Remark 6.6); and

(2) for $u, v, w \in N$ such that $v<w$, we have $\overline{u w} \neq 0$ implies $u v<u w$, and $\overline{w u} \neq 0$ implies $v u<w u$ from Lemma 6.10.

Since $B$ is graded by degree, condition (1) says that for $x_{i}, x_{j}$ from distinct cycles in $\Gamma(B)$, we have $\operatorname{deg}\left(\overline{x_{i} x_{j}}\right)=2$. If $u, w \in N$ correspond to paths in distinct cycles in $\Gamma(B)$, then Lemma 6.9 shows that $\overline{u w} \neq 0$ and $\overline{w u} \neq 0$. So condition (2) says that if $u, v, w \in N$ such that $v<w$ and $u, w$ correspond to paths in distinct cycles in $\Gamma(B)$, we have $u v<u w$ and $v u<w u$. Thus, we call any pseudopolynomial ring which satisfies (1) and (2) strictly ordered on cycles. Clearly, any pseudopolynomial ring which is almost commutative on cycles must be strictly ordered on cycles. The question is whether a pseudopolynomial ring which is strictly ordered on cycles must be almost commutative on cycles. If not, then one might ask if a pseudopolynomial ring which is strictly ordered on cycles and left Noetherian must be almost commutative on cycles.

\section{REFERENCES}

[A] J. Apel, A relationship between Gröbner bases of ideals and vector modules of G-algebras, Comtemporary Mathematics, vol. 131 (Part 2), Amer. Math. Soc., Providence, Rhode Island, 1992, pp. 195-204. MR 94f:68102

[AS] M. Artin and W. Schelter, Graded algebras of global dimension 3, Advances in Mathematics 66 (1987), 171-216. MR 88k:16003

[ATV] M. Artin, J. Tate, and M. Van Den Bergh, Some algebras associated to automorphisms of elliptic curves, The Grothendieck Festschrift, Progress in Mathematics, vol. 86, Birkhäuser, Boston, 1990, pp. 33-85. MR 92e:14002

[B] G. Bergman, The diamond lemma for ring theory, Advances in Mathematics 29 (1978), 178-218. MR 81b:16001

[Bu] B. Buchberger, An algorithm for finding a basis for the residue class ring of a zerodimensional polynomial ideal, Ph.D. Thesis, Univ. Innsbruck (Austria), 1965.

[D] L. Dickson, Finiteness of the odd perfect and primitive abundant numbers with $n$ distinct prime factors, Amer. J. Math. 35 (1913), 413-422.

[GI1] T. Gateva-Ivanova, On the Noetherianity of some associative finitely presented algebras, J. Algebra 138 (1991), 13-35. MR 92g:16031

[GI2] Noetherian properties and growth of some associative algebras, Effective Methods in Algebraic Geometry, Progress in Mathematics, vol. 94, Birkhäuser, Boston, 1991, pp. 143-158. MR 92c:16025

[GI3] Noetherian properties of skew polynomial rings with binomial relations, Trans. Amer. Math. Soc. 343 (1994), 203-219. MR 94g:16029 
[GI4] Skew Polynomial Rings with Binomial Relations, J. Algebra 185 (1996), 710-753. MR 97m:16047

[KRW] A. Kandri-Rody and V. Weispfenning, Non-commutative Gröbner bases in algebras of solvable type, J. Symbolic Computation 9 (1990), 1-26. MR 91e:13025]

[KL] G. Krause and T. Lenagan, Growth of algebras and the Gelfand-Kirillov dimension, Research Notes in Mathematics, vol. 116, Pitman Publishing Inc., Marshfield, Massachusetts, 1985. MR 86g:16001

[MR] J. McConnell and J. Robson, Noncommutative Noetherian Rings, John Wiley \& Sons, New York, 1987. MR 89j:16023

[M1] F. Mora, Groebner bases for non-commutative polynomial rings, Proc. AAECC-3, Lecture Notes in Computer Science, vol. 229, 1986, pp. 353-362. CMP 19:04

[M2] T. Mora, Groebner bases in non-commutative algebras, Proc. ISSAC 88, Lecture Notes in Computer Science, vol. 358, 1988, pp. 150-161. MR 90k:68082

[M3] S Seven variations on standard bases, preprint, Università di Genova, Dipartimento di Matematica, 1988.

[M4] An introduction to commutative and noncommutative Gröbner bases, Theoretical Computer Science 134 (1994), 131-173. MR 95i:13027

[O] J. Okniński, On monomial algebras, Archiv der Mathematik 50 (1988), 417-423. MR. 89d:16036

[SmSt] S. Smith and J. Stafford, Regularity of the four dimensional Sklyanin algebra, Compositio Mathematica 83 (1992), 259-289. MR 93h:16037

[U1] V. Ufnarovskii, A growth criterion for graphs and algebras defined by words, Math. Notes 31 (1982), 238-241.

[U2] - On the use of graphs for computing a basis, growth and Hilbert series of associative algebras, Math. USSR, Sb. 68 (2) (1991), 417-428.

Department of Mathematical Sciences, New Mexico State University, Las Cruces, New Mexico 88003

Current address: Department of Mathematics and Computer Science, Dickinson College, Carlisle, Pennsylvania 17013

E-mail address: xkramer@member.ams.org 